\title{
Rate-Splitting Multiple Access for Intelligent Reflecting Surface aided Multi-User Communications
}

\author{
Ankur Bansal, Member, IEEE, Keshav Singh, Member, IEEE, Bruno Clerckx, Senior Member, IEEE \\ Chih-Peng Li, Fellow, IEEE, and Mohamed-Slim Alouini, Fellow, IEEE
}

\begin{abstract}
Intelligent reflecting surface (IRS) has recently emerged as a promising technology for $6 \mathrm{G}$ wireless systems, due to its capability to reconfigure the wireless propagation environment. In this paper, we investigate a Rate-Splitting Multiple Access (RSMA) for IRS-assisted downlink system, where the base station (BS) communicates with single-antenna users with the help of an IRS. RSMA relies on rate-splitting (RS) at the BS and successive interference cancellation (SIC) at the users and provides a generalized multiple access framework. We derive a new architecture called IRS-RS that leverages the interplay between RS and IRS. For performance analysis, we utilize an onoff control technique to control the passive beamforming vector of the IRS-RS and derive the closed-form expressions for outage probability of cell-edge users and near users. Moreover, we also analyze the outage behavior of cell-edge users for a sufficiently large number of reflecting elements. Additionally, we also analyze the outage performance of cooperative RS based decodeand-forward (DF)-assisted framework called DF-RS. Through simulation results, it is shown that the proposed framework outperforms the corresponding DF-RS, RS without IRS and IRSassisted conventional non-orthogonal multiple access (NOMA) schemes. Furthermore, the impact of various system's parameters such as the number of IRS reflecting elements and the number of users on the system performance is revealed.
\end{abstract}

Index Terms-Intelligent reflecting surface (IRS), decode-andforward (DF), rate-splitting (RS), downlink, multi-user.

\section{INTRODUCTION}

D UE to the advancement of radio frequency (RF) microelectro-mechanical systems (MEMS) along with the plentiful applications of the reconfigurable and programmable metasurfaces [1], [2], intelligent reflecting surface (IRS) has recently attracted wide attention from both industry and academia. Multiple low-cost programmable reflecting elements are deployed at IRS, wherein each reflecting element has capability to adjust the phase of each passive antenna element. Consequently, the IRSs provide the reconfigurable reflections

Ankur Bansal is with the Department of Electrical Engineering, Indian Institute of Technology Jammu - 181 221, Jammu \& Kashmir, India. (e-mail: ankur.bansal@iitjammu.ac.in).

Keshav Singh and Chih-Peng $\mathrm{Li}$ are with the Institute of Communications Engineering, National Sun Yat-sen University, Kaohsiung 80424, Taiwan, R.O.C. (e-mail: \{keshav.singh, cpli\}@mail.nsysu.edu.tw).

Bruno Clerckx is with the Department of Electrical and Electronic Engineering, Imperial College London, London SW7 2AZ. (email: b.clerckx@imperial.ac.uk)

Mohamed-Slim Alouini is with the CEMSE Division, King Abdullah University of Science and Technology (KAUST), Thuwal, Saudi Arabia. (email: slim.alouini@kaust.edu.sa). of the impinging signals and thus, it gains huge advantages over conventional relaying protocols [3]-[5]. Moreover, [6] has pointed out IRS as one of the remedies for improving the spectrum efficiency and energy efficiency in $6 \mathrm{G}$ wireless communication network.

\section{A. Related Work}

Various schemes for IRS-assisted communications have been proposed to improve the energy and spectral efficiency [7]-[16]. In order to enhance the spectral efficiency and signal quality at the receiver, the authors in [7] have studied large intelligent surfaces (LIS)-space shift keying and LIS-spatial modulation schemes, while [8] has emphasized reconfigurable intelligent surfaces (RIS)-aided wireless networks and discussed the most important open research challenges in RIS-aided communications. An IRS-assisted nonorthogonal multiple access (NOMA) transmission scheme has been studied in [9] in order to serve more users. The authors in [10] have considered an IRS-assisted multi-user multipleinput single-output (MISO) system and proposed a robust beamforming design algorithm, whereas in [11] the achievable rate region has been identified for the coordinated IRSs-aided multi-user MISO interence channel. While [26] has focused on joint optimization of beamforming vector at the base station (BS) and the phase-shift matrix at the IRS to minimize an outage probability of IRS-assisted MISO network, resource allocation scheme has been investigated for IRS-aided MISO systems in [13]. Moreover, the authors in [14] have compared the analytical performance of IRS with conventional decodeand-forward (DF) relaying. More recently, the authors in [16] have studied the maximization of the average sum rate for two-user downlink scenario with NOMA and investigated two schemes for phase shift adjustments, while a weighted sum power minimization optimization problem has been formulated in [17] with the constraint of quality-of-service (QoS) for IRS-assisted MISO uplink network under the perfect and imperfect channel state information (CSI) knowledge. However, the works [7]-[16] have not analyzed the performance of rate-splitting multiple access (RSMA) based IRS-assisted communication network and thus, it motivates us to focus on this problem.

Recently, RSMA [18]-[25] and NOMA [9], [27] have emerged as very promising technologies which significantly improve the spectrum efficiency. Due to the flexibility of 
rate-splitting $(\mathrm{RS})$ in controlling the interference in singleinput single-output interference channels, the researchers have recently investigated the advantages of $\mathrm{RS}$ in multi-antenna MISO broadcast channels and have derived a novel multiple access framework, so-called RSMA, that encompasses Space Division Multiple Access (SDMA) and NOMA as special cases [18]-[21]. While the authors in [18] have studied the fundamental of RS and its application in massive MIMO and multi-cell scenario with respect to weighted mean square error (WMSE) and weighted sum-rate (WSR) performance metric, in [19], the sum rate maximization problem has been considered to design precoders with partial channel state information (CSI) and it has been illustrated that RS scheme boosts the achievable Degrees of Freedom (DoF). Moreover, the authors in [20] have adopted an unconventional RS transmission scheme to achieve max-min fairness in a multi-user MISO system and by utilizing the Weighted Minimum Mean Square Error (WMMSE) approach, they have highlighted that in the interference limited regimes, the RS scheme gives better performance than the conventional scheme without RS. The authors in [21] have demonstrated the advantages of RS over conventional NOMA (i.e., superposition coding with successive interference cancellation (SC-SIC)) scheme in multiantenna settings in terms of complexity and weighted sum rate performance. By considering RS and common beamforming coordination, the joint optimization of beamforming and rate allocation to maximize both energy efficiency and spectral efficiency have been studied in [23]. In [24] RS has been adopted for a multigroup multicast downlink MISO systems and a generic max-min fairness optimization problem has been formulated and solved by developing a modified WMMSE approach together with an alternating optimization algorithm and significant performance enhancements have been reported over conventional precoding techniques. However, none of the RS works in [18]-[25] have derived the analytical outage behavior of the IRS-assisted downlink system.

RS can also be applied to scenarios with relaying. The cooperative RS (CRS) has been investigated in [28]- [31]. While the weighted sum rate maximization problem has been formulated in [28] to jointly design the precoder and the resource allocation for three-node CRS network, a successive convex approximation based scheme has been proposed in [30] for jointly optimizing the precoders, message split and time slot allocation in $K$-user CRS network. However, the authors in [28]- [31] have not explored the outage performance of the CRS network.

Though the above discussions highlighted the benefits of RS in multi-antenna settings (i.e., MIMO and MISO scenarios), RS also finds benefits in single-antenna settings (i.e., each user and BS have a single antenna). Indeed, although NOMA is capacity achieving in single-input single-output (SISO) broadcast channel (BC), it is very complex since the strong user has to decode the message of $K-1$ users and therefore requires $K-1$ layers of SIC. In contrast, [21] demonstrated that in SISO BC, RS can get similar performance to NOMA but with a single SIC, therefore significantly reducing the receiver complexity. This calls for strong benefits in marrying RS with IRS even in the presence of single-antenna nodes.

\section{B. Motivation}

The above works [7]-[17] have investigated about IRSassisted communications in order to enhance the energy and spectral efficiency of MISO system, while the RS works have been explored in [18]-[25] to improve the spectrum efficiency of communication system. In addition, CRS system has been studied in [27]-[30]. Note that the works in [7][25], [27]-[30] have mainly focused on resource optimization in either IRS-assisted communication system or RS based system, however, none of the works [7]-[25], [27]-[30] have consider the joint benefit of integration of IRS and RSMA and analyzed the outage performance of RSMA based IRS-assisted communication network. Thus, it motivates us to focus on this problem. Though the works [7]-[25] have highlighted the benefits of RS in multi-antenna settings, RS also finds benefits in single-antenna settings. Indeed, although NOMA is capacity achieving in single-input single-output (SISO) broadcast channel (BC), it is very complex since the strong user has to decode the message of $K-1$ users and therefore requires $K-1$ layers of SIC. In contrast, [21] have demonstrated that in SISO BC, RS can get similar performance to NOMA but with a single SIC, therefore significantly reducing the receiver complexity. This calls for strong benefits in marrying RS with IRS even in the presence of single-antenna nodes. Motivated by the aforementioned discussions and considering the fact that IRS will play a pivotal role in the next-generation (i.e., Beyond 5G (B5G) and 6G) wireless networks as a cost, power and spectrum efficient technology, in this work, we consider a multi-user single-antenna downlink environment, where users are divided into a near users (users closer to the BS) and cell-edge users and IRSs are deployed near to cell-edge and only the cell-edge users are served by the IRSs equipped with $N$ passive reflecting elements. In order to control these $N$ passive reflecting elements, we propose an on-off technique. Moreover, we apply RS, particularly one-layer RS (1L-RS) [18], [19], [21] at the BS which splits the message of each of the $K$ users into a common and a private part. The splitted common parts of all the $K$ users are encoded into a single common stream (by using a codebook known to the users), which is decoded by all users. The splitted private part of a user is decoded only by the intended user. Accordingly, this work focuses on the outage performance analysis of the NUs and CEUs.

\section{Contributions}

The main contributions are highlighted as follows:

- We explore for the first time the interplay between RSMA and IRS and show that RSMA is a promising multiple access strategy for IRS-assisted multi-user communications. Although RSMA is a general multiple access framework [21], we emphasize on analyzing the 1L-RS for considered IRS-assisted cellular network with singleantenna BS.

- Utilizing the on-off control at the IRS, we derive the closed-form expressions for outage probability of celledge users (CEUs) and near users (NUs) by considering both common as well as private data signal-to- 
interference-plus-noise ratio (SINR). To get more insights, we study the impact of sufficiently large number of IRS reflecting elements on the outage behavior of a cell-edge user.

- In order to have a comparative analysis, we consider a cooperative RS based DF-assisted framework called DF$\mathrm{RS}$, wherein each CEU is assisted by a DF relay node. Under the DF-RS scenario, we derive the closed-form expression of outage probability for the cell-edge user with both the available and unavailable direct BS-CEU link.

- Moreover, we have compared the outage performance of the proposed IRS-RS framework with an IRS-assisted NOMA scheme [9], where a multiple-antenna BS applies NOMA in each orthogonal spatial dimension to serve a NU and a single IRS-assisted CEU. Through simulations, it is shown that the proposed IRS-RS transmission with single antenna BS provides significant performance gain as compared to the IRS-assisted NOMA framework of [9].

- Through extensive numerical results, it is shown that the proposed IRS-RS framework also outperforms the corresponding cooperative DF-RS framework and the conventional RS-based cellular network without IRS.

Structure: The rest parts of the paper is structured as follows. The system model is described in Section II. While Section III-A presents the proposed on-off scheme for controlling the passive beamforming vector at the IRS with practical phase shifts and outage analysis. The performance analysis of DF relay-assisted RS framework is illustrated in Section IV. In Section V, numerical results are presented. Finally, conclusions are drawn in Section VI.

Notations: The following notations are used throughout the paper. The lowercase and uppercase boldface letters (e.g., a and $\mathbf{A}$ ) are used to denote a vector and a matrix, respectively, while the operations of transpose, conjugate transpose, matrix inversion and element-wise conjugate are represented by $(\cdot)^{T}$, $(\cdot)^{\mathrm{H}},(\cdot)^{-1}$, and $(\cdot)^{*}$, respectively. The matrix $\mathbf{I}_{M}$ denotes an $M \times M$ identity matrix. Moreover, $\operatorname{Diag}(\cdot)$ generates a diagonal matrix with the argument on the diagonal.

\section{SySTEM MOdeL}

Consider a downlink multi-user communication system as depicted in Fig. 1 consisting of a single-antenna based BS and $K$ single-antenna users. Based on the average channel power gain, the users are divided into near users (NUs) and celledge users (CEUs) [9]. Furthermore, we assume that there is no direct link between the base station (BS) and the CEUs due to deep fading. In result, IRSs are deployed near to celledge in order to serve CEUs. Moreover, we assume that the BS and IRSs are fully coordinated with the help of a central control unit (CCU). The role of the control unit is to optimally control the reflection coefficients for obtaining optimum SINR. In order to achieve this, the control unit is assumed to be able to gather all the CSI [26]. Let $K_{1}$ and $K_{2}$ (such that $K_{1}+K_{2}=K$ ) denote the number of NUs having direct link with the BS and number of CEUs having no direct link with

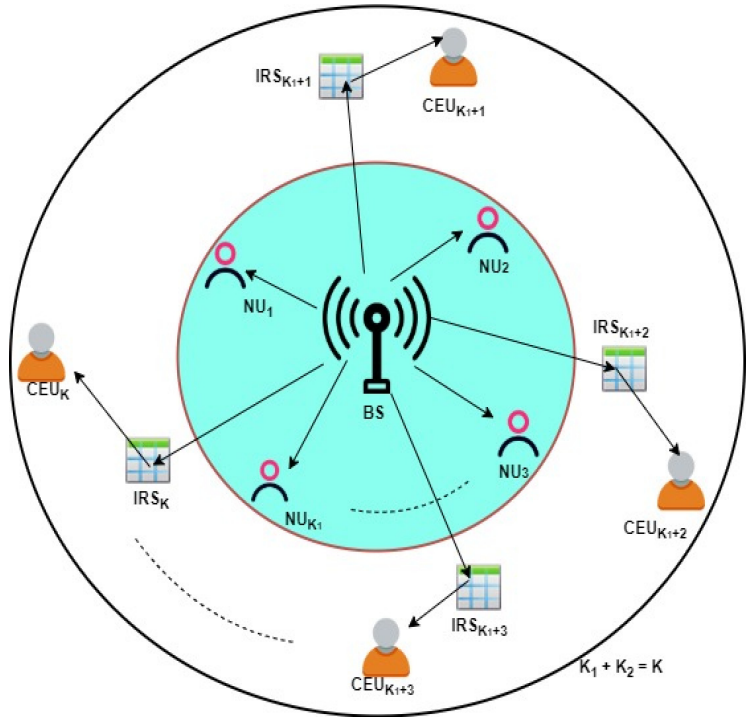

Fig. 1: An IRS-assisted downlink communication system with $K$ users and $K_{2}$ IRSs.

the BS, respectively. Moreover, the transmission of data from BS to each CEU takes place through an IRS equipped with $N$ reflecting elements. The BS utilizes the so-called 1L-RS technique of [18], [19], [21] to serve all the users.

\section{A. Transmission Protocol in $1 L-R S$}

In the considered system model, RS is applied at the BS where each user message is split into a common and a private part. The common part of each user is encoded together into a common stream using a codebook shared by all users, while private messages are encoded into the private stream for each user. To illustrate this in details, let's consider a two-user example. There are two messages $W_{1}$ and $W_{2}$ intended for user-1 and user-2, respectively. The message of each user is split into two parts, $\left\{W_{1}^{12}, W_{1}^{1}\right\}$ for user- 1 and $\left\{W_{2}^{12}, W_{2}^{2}\right\}$ for user-2. The messages $W_{1}^{12}, W_{2}^{12}$ are encoded together into a common stream $s_{12}$ using a codebook shared by both users. Hence, $s_{12}$ is a common stream required to be decoded by both users. The messages $W_{1}^{1}$ and $W_{2}^{2}$ are encoded into the private stream $s_{1}$ for user-1 and $s_{2}$ for user-2, respectively. Thus, the BS splits the $k$-th user message $\left(z_{k}\right), k=1,2, \ldots, K$ into two sub-messages known as private $\left(z_{k, p}\right)$ and common $\left(z_{k, c}\right)$ messages. The common messages of all the users are then jointly encoded into a common data symbol $x_{c}$. The private message of each user is separately encoded into a data symbol $x_{k}, k=1,2, \ldots, K$. The combined data vector at BS will be $\mathbf{x}=\left[x_{c}, x_{1}, \ldots, x_{K}\right]^{T}$. It is assumed that $\operatorname{Tr}\left[\mathbf{x x}^{\mathrm{H}}\right]=1$, where $\operatorname{Tr}[\cdot]$ is the trace of a matrix. It should be noted that $x_{c}$ will be decoded by all the users however, $x_{k}$ will only be decoded by the $k$-th user.

Let $\mathcal{P}_{B}$ is total transmit power available at the BS and $\alpha_{c}$, $\alpha_{k}$ denote the fraction of total power used for common and $k$ th private data, respectively, then the data symbol broadcasted by the BS will be $s=\sqrt{\alpha_{c} \mathcal{P}_{B}} x_{c}+\sum_{k=1}^{K} \sqrt{\alpha_{k} \mathcal{P}_{B}} x_{k}$. The coefficients $\alpha_{c}, \alpha_{k}$ are chosen such that $0 \stackrel{k=1}{\leq} \alpha_{c}, \alpha_{k} \leq 1$ and 
$\alpha_{c}+\sum_{k=1}^{K} \alpha_{k}=1$. Utilizing the $\mathrm{RS}$ at the $\mathrm{BS}$, the received signal at $\mathrm{NU}_{k_{1}}, k_{1}=1,2, \ldots, K_{1}$ can be written as

$$
y_{k_{1}}=h_{k_{1}} s+e_{k_{1}}
$$

where $h_{k_{1}} \sim \mathcal{C N}(0, \Omega)$ with $\Omega$ representing the average channel power; and $e_{k_{1}} \sim \mathcal{C N}\left(0, \sigma^{2}\right)$ denotes the noise at $\mathrm{NU}_{k_{1}}$. The use of IRSs enables BS to serve a larger number of CEUs. Similar to [9], we assume that each IRS is placed near to cell-boundary in such a way that only desired CEU can hear from it. Although, the reflected signal from a particular IRS can be treated as interference to the users associated with other IRSs, however, the interference power will be very low due to long distance and hence, we do not consider this interference at other users. Moreover, we only consider first signal reflection at IRS and completely ignores the other reflections of the same signal as they will have very low power content as compared to the first reflected signal [7]-[16]. Thus, the received signal at $\mathrm{CEU}_{k_{2}}, k_{2}=K_{1}+1, K_{1}+2, \ldots, K$ can be written as

$$
y_{k_{2}}=\mathbf{g}_{k_{2}}^{\mathrm{H}} \boldsymbol{\Phi}_{k_{2}} \mathbf{h}_{k_{2}} s+e_{k_{2}}
$$

where $\mathbf{h}_{k_{2}}$ represents the $N \times 1$ channel vector between the BS and $\mathrm{IRS}_{k_{2}}, \mathbf{g}_{k_{2}}$ denotes the channel vector between $\mathrm{IRS}_{k_{2}}$ and $\mathrm{CEU}_{k_{2}}$. Matrix $\boldsymbol{\Phi}_{k_{2}}=\operatorname{Diag}\left[\beta_{1} e^{j \phi_{1}}, \beta_{2} e^{j \phi_{2}}, \ldots, \beta_{N} e^{j \phi_{N}}\right]$ is an $N \times N$ diagonal matrix containing the reflection coefficients at $\operatorname{IRS}_{k_{2}}$, where $\beta_{n}$ and $\phi_{n}$, for $n=1,2, \ldots, N$ represents the controllable reflection amplitude and phase shift of $n$-th reflecting element. All the channel coefficients are assumed to be independent and identically distributed (i.i.d.) as $\mathcal{C N}(0,1)$; $e_{k_{2}} \sim \mathcal{C N}\left(0, \sigma^{2}\right)$ denotes the noise at $\mathrm{CEU}_{k_{2}}$. The transmit signal-to-noise ratio (SNR) can therefore be defined as $\rho_{B}=$ $\frac{\mathcal{P}_{B}}{\sigma^{2}}$. Without loss of generality, we assume $\Omega>1$ as a BS-NU link will always have more average power as compared to the BS-CEU link.

It should be noted that while the RS transmit signal model resembles a broadcasting system with unicast (private) streams and a multicast stream, the role of the common message is fundamentally different. The common message in a unicastmulticast system carries public information intended as a whole to all users in the system, while the super common message in RS encapsulates parts of private messages, and is not entirely required as by all users, although decoded by them all for interference mitigation purposes. The scenario when users don't need a common message, we simply allocate no power to common message $x_{c}$ and treat multi-user interference as noise $s=\sum_{k=1}^{K} \sqrt{\alpha_{k} \mathcal{P}_{B}} x_{k}$. In this case, the rate-splitting multiple access (RSMA) strategy resembles as a super-positioned signal transmission (SST), while it works as a Space Division Multiple Access (SDMA) when the base station has multiple antennas [18]-[21].

\section{B. SINR Characterization}

At first, all the users decode the common message $x_{c}$ by treating the interference from all private messages as noise.
Therefore, the SINR at $\mathrm{NU}_{k_{1}}$ and $\mathrm{CEU}_{k_{2}}$ for decoding the common data symbol $x_{c}$ will be given, respectively, by

$$
\begin{aligned}
\gamma_{k_{1}, c} & =\frac{\alpha_{c}\left|h_{k_{1}}\right|^{2}}{\sum_{k=1}^{K} \alpha_{k}\left|h_{k_{1}}\right|^{2}+\frac{1}{\rho_{B}}}, \\
\gamma_{k_{2}, c}= & \frac{\alpha_{c}\left|\mathbf{g}_{k_{2}}^{\mathrm{H}} \boldsymbol{\Phi}_{k_{2}} \mathbf{h}_{k_{2}}\right|^{2}}{\sum_{k=1}^{K} \alpha_{k}\left|\mathbf{g}_{k_{2}}^{\mathrm{H}} \boldsymbol{\Phi}_{k_{2}} \mathbf{h}_{k_{2}}\right|^{2}+\frac{1}{\rho_{B}}} .
\end{aligned}
$$

After successful decoding of $x_{c}$, the common message is removed from the received signals and the private message is then decoded by assuming all other private symbols as noise. Thus, the SINR for decoding the private messages of $\mathrm{NU}_{k_{1}}$ and $\mathrm{CEU}_{k_{2}}$ can be respectively written as

$$
\begin{aligned}
\gamma_{k_{1}, p} & =\frac{\alpha_{k_{1}}\left|h_{k_{1}}\right|^{2}}{\sum_{k=1 k \neq k_{1}}^{K} \alpha_{k}\left|h_{k_{1}}\right|^{2}+\frac{1}{\rho_{B}}}, \\
\gamma_{k_{2}, p} & =\frac{\alpha_{k_{2}}\left|\mathbf{g}_{k_{2}}^{\mathrm{H}} \boldsymbol{\Phi}_{k_{2}} \mathbf{h}_{k_{2}}\right|^{2}}{\sum_{k=1, k \neq k_{2}}^{K} \alpha_{k}\left|\mathbf{g}_{k_{2}}^{\mathrm{H}} \boldsymbol{\Phi}_{k_{2}} \mathbf{h}_{k_{2}}\right|^{2}+\frac{1}{\rho_{B}}}, \\
& =\frac{\sum_{k_{2}}\left|\phi_{k_{2}}^{\mathrm{H}} \mathbf{G}_{k_{2}} \mathbf{h}_{k_{2}}\right|^{2} \alpha_{k}\left|\boldsymbol{\phi}_{k_{2}}^{\mathrm{H}} \mathbf{G}_{k_{2}} \mathbf{h}_{k_{2}}\right|^{2}+\frac{1}{\rho_{B}}}{\sum_{k=1, k \neq k_{2}}^{K}}
\end{aligned}
$$

where vector $\phi_{k_{2}}^{\mathrm{H}}$ is a row vector of size $1 \times N$ containing main diagonal elements of matrix $\boldsymbol{\Phi}_{k_{2}}$. The matrix $\mathbf{G}_{k_{2}}$ is an $N \times N$ diagonal matrix with diagonal elements obtained from $\mathbf{g}_{k_{2}}^{\mathrm{H}}$.

\section{Performance Analysis of IRS-Assisted RS}

In order to analyze the performance of IRS-assisted RS framework for multi-user downlink communication system, it is important to choose the IRS beamforming matrix $\boldsymbol{\Phi}_{k_{2}}$ or equivalently, vector $\phi_{k_{2}}^{\mathrm{H}}$. There are many design solutions such as Zero Forcing (ZF) beamforming (an ideal solution that require infinite resolution), discrete fourier transform (DFT) based beamforming (a finite resolution based design), and an on-off control etc. However, due to low-cost implementation for controlling the IRS-RS, we consider on-off control method [9].

\section{A. On-Off Technique to Control Passive Beamforming of IRS}

In on-off controlling, it is assumed that the elements of beamforming vector $\phi_{k_{2}}^{\mathrm{H}}$ are either 1 (on) or 0 (off). Let us define a matrix $\mathbf{F}=\mathbf{I}_{L} \otimes \mathbf{v}, L \in \mathbb{I}^{+}$, where $\mathbb{I}^{+}$represents the set of positive integers and $\mathbf{v}=\left\{v_{d}\right\}_{d=1}^{D}$ is a unit norm row vector of length $D, D \in \mathbb{I}^{+}$such that $N=L D$ and $v_{d}=\frac{1}{\sqrt{D}}$; and $\otimes$ denotes the Kronecker product. It can be noticed that all the row vectors in $\mathbf{F}$ are orthonormal vectors. Vector $\phi_{k_{2}}^{\mathrm{H}}$ is selected as $\ell$-th row vector $\mathbf{f}_{\ell}, \ell=1,2, \ldots, L$ of $\mathbf{F}$ such 
that $\mathbf{f}_{\ell}$ maximizes the SINRs at CEU given in (4) and (6). Therefore, the SINRs at CEU are evaluated as

$$
\begin{gathered}
\tilde{\gamma}_{k_{2}, c} \triangleq \max \left(\left\{\gamma_{k_{2}, c}\left(\mathbf{f}_{\ell}\right)\right\}_{\ell=1}^{L}\right), \\
\tilde{\gamma}_{k_{2}, p} \triangleq \max \left(\left\{\gamma_{k_{2}, p}\left(\mathbf{f}_{\ell}\right)\right\}_{\ell=1}^{L}\right),
\end{gathered}
$$

where

$$
\begin{aligned}
\gamma_{k_{2}, c}\left(\mathbf{f}_{\ell}\right) & =\frac{\alpha_{c}\left|\mathbf{f}_{\ell} \mathbf{G}_{k_{2}} \mathbf{h}_{k_{2}}\right|^{2}}{\sum_{k=1}^{K} \alpha_{k}\left|\mathbf{f}_{\ell} \mathbf{G}_{k_{2}} \mathbf{h}_{k_{2}}\right|^{2}+\frac{1}{\rho_{B}}}, \\
\gamma_{k_{2}, p}\left(\mathbf{f}_{\ell}\right) & =\frac{\alpha_{k_{2}}\left|\mathbf{f}_{\ell} \mathbf{G}_{k_{2}} \mathbf{h}_{k_{2}}\right|^{2}}{\sum_{k=1, k \neq k_{2}}^{K} \alpha_{k}\left|\mathbf{f}_{\ell} \mathbf{G}_{k_{2}} \mathbf{h}_{k_{2}}\right|^{2}+\frac{1}{\rho_{B}}} .
\end{aligned}
$$

Observation 1: It should be noted that random variables (RVs) $\gamma_{k_{2}, c}\left(\mathbf{f}_{1}\right), \gamma_{k_{2}, c}\left(\mathbf{f}_{2}\right), \ldots, \gamma_{k_{2}, c}\left(\mathbf{f}_{L}\right)$ are independent to each other. Similarly, the instantaneous SINRs $\gamma_{k_{2}, p}\left(\mathbf{f}_{1}\right), \gamma_{k_{2}, p}\left(\mathbf{f}_{2}\right), \ldots, \gamma_{k_{2}, p}\left(\mathbf{f}_{L}\right)$ are also independent to each other. Furthermore, the RV $\gamma_{k_{2}, c}\left(\mathbf{f}_{\ell}\right)$ is dependent on $\gamma_{k_{2}, p}\left(\mathbf{f}_{\ell}\right)$, for all $\ell$. However, the pair $\left\{\gamma_{k_{2}, c}\left(\mathbf{f}_{i}\right), \gamma_{k_{2}, p}\left(\mathbf{f}_{i}\right)\right\}$ remains independent of the pair $\left\{\gamma_{k_{2}, c}\left(\mathbf{f}_{j}\right), \gamma_{k_{2}, p}\left(\mathbf{f}_{j}\right)\right\}$, for $i \neq j, i, j=$ $1,2, \ldots, L$.

We have numerically and analytically verified that a vector $\mathbf{f}_{\ell}$ that maximizes the common SINR at CEU also maximizes the private SINR of that CEU. The analytical proof for the same is given in Appendix A.

\section{B. Outage Analysis for CEUs}

A CEU will not suffer outage if and only if the SINR values for common and private symbols are simultaneously more than the corresponding threshold SINRs (equivalently, the targeted quality-of-service (QoS)). Hence, the outage probability at $\mathrm{CEU}_{k_{2}}$ is defined as,

$$
\mathbb{P}_{k_{2}}^{\text {out }}=1-\operatorname{Pr}\left\{\tilde{\gamma}_{k_{2}, c}>\tau_{c}, \tilde{\gamma}_{k_{2}, p}>\tau_{p}\right\},
$$

where $\tau_{c}=2^{\bar{R}_{c}}-1$ and $\tau_{p}=2^{\bar{R}_{p}}-1$ are threshold SINRs for common and private messages, respectively, with $\bar{R}_{c}$ and $\bar{R}_{p}$ being their targeted data rates, respectively. $\operatorname{Pr}\{\cdot, \cdot\}$ represents the joint probability. Using the concepts of joint probabilities [32], the outage probability in (9) can be further simplified as,

$$
\mathbb{P}_{k_{2}}^{\text {out }}=\mathbb{F}_{\tilde{\gamma}_{k_{2}, c}}\left(\tau_{c}\right)+\mathbb{F}_{\tilde{\gamma}_{k_{2}, p}}\left(\tau_{p}\right)-\mathbb{F}_{\tilde{\gamma}_{k_{2}, c}, \tilde{\gamma}_{k_{2}, p}}\left(\tau_{c}, \tau_{p}\right),
$$

where $\mathbb{F}_{X}(\cdot)$ is the marginal cumulative distribution function (CDF) of RV $X$ and $\mathbb{F}_{X, Y}(\cdot, \cdot)$ is the joint CDF of $X$ and $Y$. Following the discussion in Observation 1, we can rewrite the outage probability in (10) as

$$
\begin{array}{r}
\mathbb{P}_{k_{2}}^{\text {out }}=\prod_{\ell=1}^{L} \mathbb{F}_{\gamma_{k_{2}, c}\left(\mathbf{f}_{\ell}\right)}\left(\tau_{c}\right)+\prod_{\ell=1}^{L} \mathbb{F}_{\gamma_{k_{2}, p}\left(\mathbf{f}_{\ell}\right)}\left(\tau_{p}\right) \\
-\prod_{\ell=1}^{L} \mathbb{F}_{\gamma_{k_{2}, c}\left(\mathbf{f}_{\ell}\right), \gamma_{k_{2}, p}\left(\mathbf{f}_{\ell}\right)}\left(\tau_{c}, \tau_{p}\right) .
\end{array}
$$

If marginal and joint statistics of $\gamma_{k_{2}, c}\left(\mathbf{f}_{\ell}\right)$ and $\gamma_{k_{2}, p}\left(\mathbf{f}_{\ell}\right)$ are same for all $\ell$, the outage probability in (11) can be given as

$$
\begin{aligned}
\mathbb{P}_{k_{2}}^{\text {out }}=\left[\mathbb{F}_{\gamma_{k_{2}, c}\left(\mathbf{f}_{\ell}\right)}\left(\tau_{c}\right)\right]^{L}+\left[\mathbb{F}_{\gamma_{k_{2}, p}\left(\mathbf{f}_{\ell}\right)}\left(\tau_{p}\right)\right]^{L} \\
-\left[\mathbb{F}_{\gamma_{k_{2}, c}\left(\mathbf{f}_{\ell}\right), \gamma_{k_{2}, p}\left(\mathbf{f}_{\ell}\right)}\left(\tau_{c}, \tau_{p}\right)\right]^{L} .
\end{aligned}
$$

Lemma 1: For an arbitrary CEU in multi-user IRS-assisted RS technique, the joint CDF of common and private SINRs for a fixed passive beamforming vector $\mathbf{f}_{\ell}$, is given as

$$
\begin{aligned}
& \mathbb{F}_{\gamma_{k_{2}, c}\left(\mathbf{f}_{\ell}\right), \gamma_{k_{2}, p}\left(\mathbf{f}_{\ell}\right)}\left(\tau_{c}, \tau_{p}\right)= \\
& 1-\frac{2}{\Gamma(D)}\left(\frac{D \tau}{\rho_{B}}\right)^{\frac{D}{2}} K_{D}\left(2 \sqrt{\frac{D \tau}{\rho_{B}}}\right),
\end{aligned}
$$

where $K_{v}(\cdot)$ is the $v$-th order modified Bessel's function of second kind, $\Gamma(\cdot)$ is the Gamma function, and $\tau>0$ is given as $\tau=\min \left(\delta_{1}\left(\tau_{c}\right), \delta_{2}\left(\tau_{p}\right)\right)$ with

$$
\delta_{1}\left(\tau_{c}\right)=\frac{\tau_{c}}{\alpha_{c}-\tau_{c} \sum_{k=1}^{K} \alpha_{k}}, \quad \delta_{2}\left(\tau_{p}\right)=\frac{\tau_{p}}{\alpha_{k_{2}}-\tau_{p} \sum_{k=1, k \neq k_{2}}^{K} \alpha_{k}} .
$$

Proof: See Appendix B for the proof.

Corollary 1: The marginal CDFs $\mathbb{F}_{\gamma_{k_{2}, c}\left(\mathbf{f}_{\ell}\right)}\left(\tau_{c}\right)$ and $\mathbb{F}_{\gamma_{k_{2}, p}\left(\mathbf{f}_{\ell}\right)}\left(\tau_{p}\right)$ can be obtained by using $\tau_{c} \rightarrow \infty$ and $\tau_{p} \rightarrow \infty$ in the joint CDF expression given in (13), as

$$
\begin{aligned}
\mathbb{F}_{\gamma_{k_{2}, c}\left(\mathbf{f}_{\ell}\right)}\left(\tau_{c}\right) & = \\
1 & -\frac{2}{\Gamma(D)}\left(\frac{D \delta_{1}\left(\tau_{c}\right)}{\rho_{B}}\right)^{\frac{D}{2}} K_{D}\left(2 \sqrt{\frac{D \delta_{1}\left(\tau_{c}\right)}{\rho_{B}}}\right), \\
\mathbb{F}_{\gamma_{k_{2}, p}\left(\mathbf{f}_{\ell}\right)}\left(\tau_{p}\right) & = \\
1 & -\frac{2}{\Gamma(D)}\left(\frac{D \delta_{2}\left(\tau_{p}\right)}{\rho_{B}}\right)^{\frac{D}{2}} K_{D}\left(2 \sqrt{\frac{D \delta_{2}\left(\tau_{p}\right)}{\rho_{B}}}\right) .
\end{aligned}
$$

The overall outage probability of $\mathrm{CEU}_{k_{2}}$ can thus be evaluated by using (13)-(16) in (12). It is shown through numerical results that the choice of $L=N$ (or $D=1$ ) gives the best outage performance for all the values of BS transmit power. The intuitive reason for the same and the information regarding which IRS elements are turned on are given in the following remarks.

Remark 1: It should be noted that the on-off control at IRS does not considers all possible combinations of selecting $D$ elements out of $N$ (i.e., $\left(\begin{array}{l}N \\ D\end{array}\right)$ ), however, it only focuses on $\frac{N}{D}=L$ disjoint subsets of reflecting elements, each with cardinality $D$. Thus, the random variable $Z_{\ell}, \ell=1,2, \ldots, L$ (defined before (49)) is a sum of $D$ i.i.d. random variables (refer (42)). Intuitively, the sum of $D$ i.i.d. random variables results in similar statistical advantages as achieved for single random variable (i.e., $D=1$ ). Therefore, we do not get any diversity benefits for $D>1$ as the SNR is proportional to $\left|Z_{\ell}\right|^{2}$. Moreover, obtaining the maximum of $L$ independent SNRs (as defined in (7)) always provides the best results for the largest possible $L$ (i.e., $L=N$ ). Therefore, the on-off control at IRS for RS based BS gives the best results for $L=N($ or $D=1)$.

Remark 2: To identify which $D$ elements should be turned on in general on-off scenario, an index should be assigned to all the reflecting elements and $L$ non-overlapping sets of $D$ reflecting elements should be formed. A set having the 
maximum sum of its elements will give the information about active reflecting elements, i.e., all those reflecting elements, whose index are present in the set, will be turned on provided that the sum of composite BS-IRS-CEU channel gains for those elements is maximum among all possible disjoint sets.

Observation 2: It can be observed from (14) that for given $\tau_{c}$ and $\tau_{p}$ (equivalently, $\bar{R}_{c}$ and $\bar{R}_{p}$ ), there is always a constraint for $\alpha_{c}$ and $\alpha_{k_{2}}$ as

$$
\alpha_{c}>\frac{\tau_{c}}{1+\tau_{c}}, \quad \text { and } \quad \alpha_{k_{2}}>\frac{\tau_{p}\left(1-\alpha_{c}\right)}{1+\tau_{p}},
$$

such that $\delta_{1}\left(\tau_{c}\right)>0$ and $\delta_{2}\left(\tau_{p}\right)>0$. From the valid range of $\alpha_{c}$ and $\alpha_{k_{2}}$, we can choose the optimum values of these power allocation coefficients for which the outage probability of a CEU is minimized. Moreover, the condition for $\alpha_{k_{1}}$ will be same as that for $\alpha_{k_{2}}$ provided $\tau_{p}$ is same for both NU and CEU.

In Figs. 2 (a) and (b), we have considered 2-user scenario with single NU and CEU and have shown the outage probability variations of the CEU for varying $\alpha_{c}$ and $\alpha_{2}$. Initially, we set the target data rate for common and private messages as $\bar{R}_{c}=$ $\bar{R}_{p}=0.25$ bits per channel use (BPCU). We also assume that out of the total available power for private messages of all the users, $60 \%$ power is allocated to the CEU, i.e., $\alpha_{2}=$ $0.6\left(1-\alpha_{c}\right)$. Under these settings, it can be observed from Fig. 2(a) that (i) the valid range for $\alpha_{c}$ is $0.17<\alpha_{c}<1$, and (ii) the outage probability is minimized at $\alpha_{c} \approx 0.4$. However, if we increase the target data rate $\bar{R}_{c}$ to $0.5 \mathrm{BPCU}$ (by keeping $\bar{R}_{p}$ fixed), we observe an increase in the optimum value of $\alpha_{c}$ which minimizes the outage probability of the CEU. In Fig. 2(b), we have used $\alpha_{c}=0.4$ and varied $\alpha_{2}$ from 0 to $\left(1-\alpha_{c}\right)$. For $\bar{R}_{c}=\bar{R}_{c}=0.25 \mathrm{BPCU}$, it can be noticed from Fig. 2(b) that (i) $0.1<\alpha_{2}<0.6$, and (ii) the outage probability of CEU is minimum at $\alpha_{2} \approx 0.36$, which is $60 \%$ of $\left(1-\alpha_{c}\right)$. Further, on raising the target data rate $\bar{R}_{p}$ for the $\mathrm{CEU}$, the optimum power allocation coefficient for the CEU increases accordingly.

In the numerical results section, the optimum values of power allocation coefficients (for given target data rates of common and private data) are chosen in similar fashion as described in Observation 2.

\section{Asymptotic Analysis for large $N$}

To obtain further insights, we investigate the outage behavior of IRS-assisted CEU for sufficiently high reflecting elements, i.e., $N \rightarrow \infty$.

Lemma 2: For a multi-user downlink network employing 1L-RS scheme, the joint CDF of optimum common and private SINRs at an arbitrary IRS-assisted CEU can be given for large number of reflecting elements as,

$$
\begin{aligned}
& \mathcal{F}_{\tilde{\gamma}_{k_{2}, c}, \tilde{\gamma}_{k_{2}, p}}\left(\tau_{c}, \tau_{p}\right)=\lim _{N \rightarrow \infty} \mathbb{F}_{\tilde{\gamma}_{k_{2}, c}, \tilde{\gamma}_{k_{2}, p}}\left(\tau_{c}, \tau_{p}\right) \\
&=\exp \left\{-\frac{2 N}{\Gamma(D+1)}\left(\frac{D \tau}{\rho_{B}}\right)^{\frac{D}{2}} K_{D}\left(2 \sqrt{\frac{D \tau}{\rho_{B}}}\right)\right\},
\end{aligned}
$$

where $\tau=\min \left(\delta_{1}\left(\tau_{c}\right), \delta_{2}\left(\tau_{p}\right)\right)$ with $\delta_{1}\left(\tau_{c}\right)$ and $\delta_{2}\left(\tau_{p}\right)$ are given in (14).

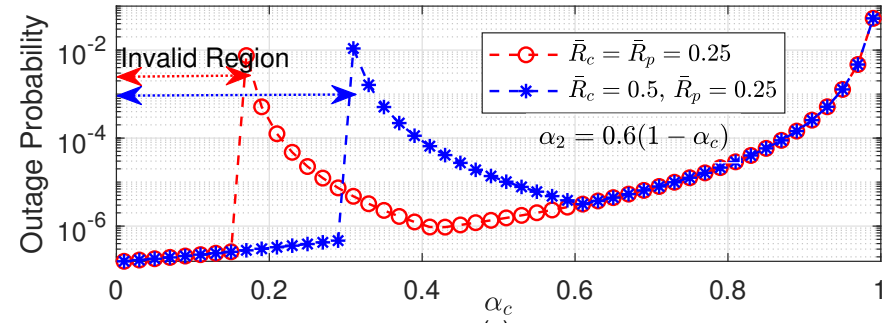

(a)

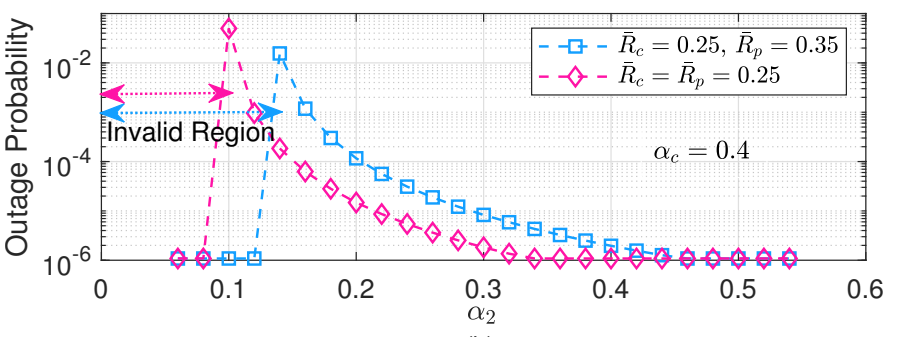

(b)

Fig. 2: Outage performance of CEU with varying $\alpha_{c}$ and $\alpha_{2}$ for $K=2$ and $N=4$.

Proof: Following (10)-(13), we can write the joint CDF of $\tilde{\gamma}_{k_{2}, c}$ and $\tilde{\gamma}_{k_{2}, p}$ as

$$
\begin{aligned}
\mathbb{F}_{\tilde{\gamma}_{k_{2}, c}, \tilde{\gamma}_{k_{2}, p}}\left(\tau_{c}, \tau_{p}\right)= & \\
& {\left[1-\frac{2}{\Gamma(D)}\left(\frac{D \tau}{\rho_{B}}\right)^{\frac{D}{2}} K_{D}\left(2 \sqrt{\frac{D \tau}{\rho_{B}}}\right)\right]^{L} . }
\end{aligned}
$$

For sufficiently large number of reflecting elements (i.e., $N \rightarrow \infty$ ), we can have $L \rightarrow \infty$ provided $D$ is fixed. Using the standard approximation $\lim _{n \rightarrow \infty}(1-x)^{n} \approx e^{-n x}$ with some algebra, we obtain (18).

Corollary 2: For asymptotically high $N$, the marginal CDFs $\mathbb{F}_{\tilde{\gamma}_{k_{2}, c}}\left(\tau_{c}\right)$ and $\mathbb{F}_{\tilde{\gamma}_{k_{2}, p}}\left(\tau_{p}\right)$ can be obtained by using $\tau_{c} \rightarrow \infty$ and $\tau_{p} \rightarrow \infty$ in the asymptotic joint $\mathrm{CDF}$ expression given in (18), as

$$
\begin{aligned}
& \mathcal{F}_{\tilde{\gamma}_{k_{2}, a}}\left(\tau_{a}\right)=\lim _{N \rightarrow \infty} \mathbb{F}_{\tilde{\gamma}_{k_{2}, a}}\left(\tau_{a}\right) \\
& =\exp \left\{-\frac{2 N}{\Gamma(D+1)}\left(\frac{D \delta_{i}\left(\tau_{a}\right)}{\rho_{B}}\right)^{\frac{D}{2}} K_{D}\left(2 \sqrt{\frac{D \delta_{i}\left(\tau_{a}\right)}{\rho_{B}}}\right)\right\},
\end{aligned}
$$

where $(i, a) \in\{(1, c),(2, p)\}$.

Utilizing (18) and (20) in (10), we can evaluate the asymptotic outage probability of $\mathrm{CEU}_{k_{2}}$ for $N \rightarrow \infty$ as

$$
\begin{aligned}
\mathcal{P}_{k_{2}}^{\text {out }} & =\lim _{N \rightarrow \infty} \mathbb{P}_{k_{2}}^{\text {out }} \\
& =\mathcal{F}_{\tilde{\gamma}_{k_{2}, c}}\left(\tau_{c}\right)+\mathcal{F}_{\tilde{\gamma}_{k_{2}, p}}\left(\tau_{p}\right)-\mathcal{F}_{\tilde{\gamma}_{k_{2}, c}, \tilde{\gamma}_{k_{2}, p}}\left(\tau_{c}, \tau_{p}\right),
\end{aligned}
$$

We introduce a performance metric known as Log-Asymptotic Outage Order for an arbitrary $\mathrm{CEU}_{k_{2}}$, denoted by $\mathcal{O}_{k_{2}, N}$. It is defined as the negative ratio of natural logarithm of asymptotic outage probability (for large $N$ ) of $\mathrm{CEU}_{k_{2}}$ and number of reflecting elements $N$, i.e.,

$$
\mathcal{O}_{k_{2}, N}=-\frac{1}{N} \ln \mathcal{P}_{k_{2}}^{\text {out }},
$$




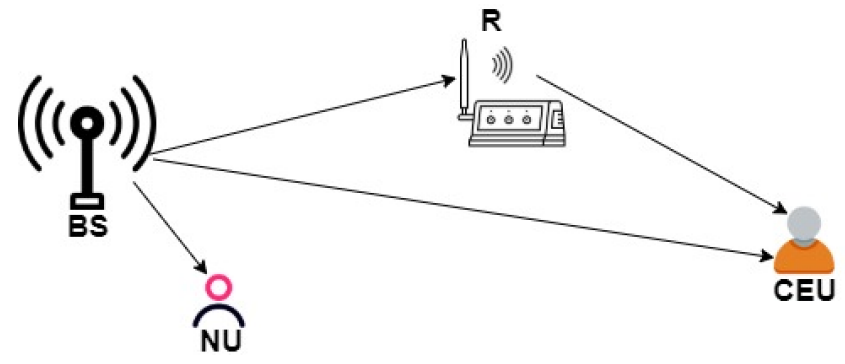

Fig. 3: A DF-RS downlink communication system showing a single CEU assisted with single DF relay.

where $\mathcal{P}_{k_{2}}^{\text {out }}$ is given in (21). The insights obtained from the Log-Asymptotic Outage Order are discussed in the numerical results section.

\section{Outage Analysis for NUs}

For NUs, the outage probability can be defined as

$$
\begin{aligned}
\mathbb{P}_{k_{1}}^{\text {out }} & =1-\operatorname{Pr}\left\{\gamma_{k_{1}, c}>\tau_{c}, \gamma_{k_{1}, p}>\tau_{p}\right\}, \\
& =\mathbb{F}_{\gamma_{k_{1}, c}}\left(\tau_{c}\right)+\mathbb{F}_{\gamma_{k_{1}, p}}\left(\tau_{p}\right)-\mathbb{F}_{\gamma_{k_{1}, c}, \gamma_{k_{1}, p}}\left(\tau_{c}, \tau_{p}\right) .
\end{aligned}
$$

Since $h_{k_{1}} \sim \mathcal{C N}(0, \Omega)$, we can write that $\left|h_{k_{1}}\right|^{2} \sim \operatorname{Exp}(\Omega)$. After some simple mathematical manipulations, we get

$$
\begin{aligned}
\mathbb{F}_{\gamma_{k_{1}, c}}\left(\tau_{c}\right) & =1-\exp \left(-\frac{\delta_{1}\left(\tau_{c}\right)}{\Omega \rho_{B}}\right) \\
\mathbb{F}_{\gamma_{k_{1}, p}}\left(\tau_{p}\right) & =1-\exp \left(-\frac{\delta_{2}\left(\tau_{p}\right)}{\Omega \rho_{B}}\right) \\
\mathbb{F}_{\gamma_{k_{1}, c}, \gamma_{k_{1}, p}}\left(\tau_{c}, \tau_{p}\right) & =1-\exp \left(-\frac{\min \left(\delta_{1}\left(\tau_{c}\right), \delta_{2}\left(\tau_{p}\right)\right)}{\Omega \rho_{B}}\right)
\end{aligned}
$$

Using (24)-(26) in (23), we get the outage probability of a NU.

\section{Performance Analysis of DF-RS Framework}

In order to compare the performance of IRS-RS framework with a DF-RS network, we analyze the outage performance of the considered multi-user downlink communication system by replacing the IRS I $_{2}$ with a single-antenna based DF relay $\mathrm{R}_{k_{2}}$ as shown in Fig. 3. Contrary to the cooperative RS models considered in [28] and [30], we assume that the relay $\mathrm{R}_{k_{2}}$ does not receive its own data from $\mathrm{BS}$. It completely decodes the common message followed by the decoding of private message of $\mathrm{CEU}_{k_{2}}$, received from BS and forwards them to $\mathrm{CEU}_{k_{2}}$ only by utilizing RS. In the following subsections, we consider two different scenarios based on the availability of direct BS-CEU link and obtain the outage probability under both scenarios.

\section{A. With Direct BS-CEU Link}

We assume that each CEU is served directly by the BS as well as through a DF-based cooperative link as shown in Fig. 3. Let $h_{0, k_{2}} \sim \mathcal{C N}\left(0, \Omega^{\prime}\right)$ and $h_{1, k_{2}} \sim \mathcal{C N}(0,1)$ represent the channel coefficients of direct BS-CEU $k_{2}$ link (with average power $\Omega^{\prime}$ ) and the $B S-R_{k_{2}}$ link, respectively. Since the BS has a larger separation from the CEU as compared to the NU, the average channel power of BS-CEU link is assumed to be smaller than that of the BS-NU link, (i.e., $\Omega^{\prime}<\Omega$ ). Thus, the common and private message SINRs at $\mathrm{CEU}_{k_{2}}$ and $\mathrm{R}_{k_{2}}$ will be given as

$$
\begin{aligned}
\gamma_{i, k_{2}, c} & =\frac{\alpha_{c}\left|h_{i, k_{2}}\right|^{2}}{\sum_{k=1}^{K} \alpha_{k}\left|h_{i, k_{2}}\right|^{2}+\frac{1}{\rho_{B}}}, \\
\gamma_{i, k_{2}, p} & =\frac{\alpha_{k_{2}}\left|h_{i, k_{2}}\right|^{2}}{\sum_{k=1, k \neq k_{2}}^{K} \alpha_{k}\left|h_{i, k_{2}}\right|^{2}+\frac{1}{\rho_{B}}},
\end{aligned}
$$

where $i=0,1$ corresponds to $\mathrm{BS}-\mathrm{CEU}_{k_{2}}$ and $\mathrm{BS}-\mathrm{R}_{k_{2}}$ links, respectively. The $k_{2}$-th relay node employs $\mathrm{RS}$ with the decoded common data stream and one private data streams (for $\mathrm{CEU}_{k_{2}}$ ) and forwards the combined data stream to $\mathrm{CEU}_{k_{2}}$ after re-encoding. If the channel coefficient of the $k_{2}$-th $\mathrm{R}$ CEU link is denoted by $h_{2, k_{2}} \sim \mathcal{C N}(0,1)$, then we have

$$
\begin{aligned}
\gamma_{2, k_{2}, c} & =\frac{\hat{\alpha}_{c}\left|h_{2, k_{2}}\right|^{2}}{\hat{\alpha}_{k_{2}}\left|h_{2, k_{2}}\right|^{2}+\frac{1}{\rho_{R}}}, \\
\gamma_{2, k_{2}, p} & =\hat{\alpha}_{k_{2}}\left|h_{2, k_{2}}\right|^{2} \rho_{R},
\end{aligned}
$$

where $\hat{\alpha}_{c}$ and $\hat{\alpha}_{k_{2}}$ are the fraction of the relay transmit power $\left(\mathcal{P}_{R}\right)$ associated with common and $k_{2}$-th private data streams, respectively, such that $\hat{\alpha}_{c}+\hat{\alpha}_{k_{2}}=1$, and $\rho_{R}=\frac{\mathcal{P}_{R}}{\sigma^{2}}$ is the transmit SNR by the relay. Utilizing the concepts of a DFbased cooperative relay network with direct link, the end-toend SINRs of common and private data streams at $\mathrm{CEU}_{k_{2}}$ can be written as

$$
\begin{aligned}
& \check{\gamma}_{k_{2}, c}=\gamma_{0, k_{2}, c}+\min \left(\gamma_{1, k_{2}, c}, \gamma_{2, k_{2}, c}\right), \\
& \check{\gamma}_{k_{2}, p}=\gamma_{0, k_{2}, p}+\min \left(\gamma_{1, k_{2}, p}, \gamma_{2, k_{2}, p}\right),
\end{aligned}
$$

and the outage probability of $\mathrm{CEU}_{k_{2}}$ under the considered DF-RS cooperative scenario can be obtained as

$$
\begin{aligned}
\mathbb{P}_{k_{2}}^{\text {out,DF }} & =1-\operatorname{Pr}\left\{\check{\gamma}_{k_{2}, c}>\tau_{c}, \check{\gamma}_{k_{2}, p}>\tau_{p}\right\} \\
& \triangleq 1-\mathcal{I} .
\end{aligned}
$$

Utilizing (29) in (30), we can write

$$
\begin{gathered}
\mathcal{I}=\operatorname{Pr}\left\{\gamma_{0, k_{2}, c}+\min \left(\gamma_{1, k_{2}, c}, \gamma_{2, k_{2}, c}\right)>\tau_{c},\right. \\
\left.\gamma_{0, k_{2}, p}+\min \left(\gamma_{1, k_{2}, p}, \gamma_{2, k_{2}, p}\right)>\tau_{p}\right\}, \\
=\operatorname{Pr}\left\{\gamma_{0, k_{2}, c}+\gamma_{1, k_{2}, c}>\tau_{c}, \gamma_{0, k_{2}, c}+\gamma_{2, k_{2}, c}>\tau_{c},\right. \\
\left.\gamma_{0, k_{2}, p}+\gamma_{1, k_{2}, p}>\tau_{p}, \gamma_{0, k_{2}, p}+\gamma_{2, k_{2}, p}>\tau_{p}\right\} .
\end{gathered}
$$

If we assume that $\gamma_{0, k_{2}, c}$ and $\gamma_{0, k_{2}, p}$ are known, then the probability term in (31) can be conditionally written as

$$
\begin{aligned}
\mathcal{I}\left(\gamma_{0, k_{2}, c}\right. & \left.=\mu, \gamma_{0, k_{2}, p}=\nu\right), \\
& =\operatorname{Pr}\left\{\gamma_{1, k_{2}, c}>\tau_{c}-\mu, \gamma_{1, k_{2}, p}>\tau_{p}-\nu\right\}, \\
& \times \operatorname{Pr}\left\{\gamma_{2, k_{2}, c}>\tau_{c}-\mu, \gamma_{2, k_{2}, p}>\tau_{p}-\nu\right\} .
\end{aligned}
$$

Utilizing (27) in (32) along with the use of $\left|h_{1, k_{2}}\right|^{2} \sim \operatorname{Exp}(1)$, we get

$$
\begin{aligned}
\operatorname{Pr}\left\{\gamma_{1, k_{2}, c}\right. & \left.>\tau_{c}-\mu, \gamma_{1, k_{2}, p}>\tau_{p}-\nu\right\}, \\
& =\exp \left[-\frac{\max \left(\delta_{1}\left(\tau_{c}-\mu\right), \delta_{2}\left(\tau_{p}-\nu\right)\right)}{\rho_{B}}\right],
\end{aligned}
$$


where $\delta_{1}(\cdot)$ and $\delta_{2}(\cdot)$ are defined in (14). Similarly, we can write

$$
\begin{aligned}
\operatorname{Pr}\left\{\gamma_{2, k_{2}, c}\right. & \left.>\tau_{c}-\mu, \gamma_{2, k_{2}, p}>\tau_{p}-\nu\right\} \\
& =\exp \left[-\frac{\max \left(\delta_{3}\left(\tau_{c}-\mu\right), \delta_{4}\left(\tau_{p}-\nu\right)\right)}{\rho_{R}}\right],
\end{aligned}
$$

where

$$
\delta_{3}\left(\tau_{c}-\mu\right)=\frac{\tau_{c}-\mu}{\hat{\alpha}_{c}-\left(\tau_{c}-\mu\right) \hat{\alpha}_{k_{2}}}, \quad \delta_{4}\left(\tau_{p}-\nu\right)=\frac{\tau_{p}-\nu}{\hat{\alpha}_{k_{2}}} .
$$

Substituting (33) and (34) in (32) and jointly averaging over $\gamma_{0, k_{2}, c}$ and $\gamma_{0, k_{2}, p}$, we can evaluate (31) as

$$
\begin{aligned}
\mathcal{I}=\int_{0}^{\infty} \int_{0}^{\infty} & \mathcal{I}\left(\gamma_{0, k_{2}, c}=\mu, \gamma_{0, k_{2}, p}=\nu\right) \\
& \times f_{\gamma_{0, k_{2}, c}, \gamma_{0, k_{2}, p}}(\mu, \nu) d \mu d \nu,
\end{aligned}
$$

where $f_{\gamma_{0, k_{2}, c}, \gamma_{0, k_{2}, p}}(\mu, \nu)$ is the joint PDF of common and private SINRs at $\mathrm{CEU}_{k_{2}}$ received through the direct link from BS, and can be obtained as

$$
f_{\gamma_{0, k_{2}, c}, \gamma_{0, k_{2}, p}}(\mu, \nu)=\frac{\partial^{2}}{\partial \mu \partial \nu} \mathbb{F}_{\gamma_{0, k_{2}, c}, \gamma_{0, k_{2}, p}}(\mu, \nu) .
$$

The joint $\operatorname{CDF} \mathbb{F}_{\gamma_{0, k_{2}, c}, \gamma_{0, k_{2}, p}}(\mu, \nu)$ can be evaluated from (26) by replacing $\Omega$ with $\Omega^{\prime}$ and $\left(\tau_{c}, \tau_{p}\right)$ with $(\mu, \nu)$. Hence, the joint PDF in (37) will be

$$
\begin{aligned}
f_{\gamma_{0, k_{2}, c}, \gamma_{0, k_{2}, p}}(\mu, \nu) & =\frac{1}{\Omega^{\prime} \rho_{B}} \exp \left[-\frac{\min \left(\delta_{1}(\mu), \delta_{2}(\nu)\right)}{\Omega^{\prime} \rho_{B}}\right] \\
& \times \frac{\partial^{2}}{\partial \mu \partial \nu}\left[\min \left(\delta_{1}(\mu), \delta_{2}(\nu)\right)\right] .
\end{aligned}
$$

Using (36)-(38), we numerically obtain the outage probability values in (30).

\section{B. Without Direct BS-CEU Link}

If we assume that the direct path between the BS and the CEU is not available due to high rise buildings and other obstacles, then the end-to-end SINRs of common and private data streams at $\mathrm{CEU}_{k_{2}}$ (given in (29)) can be re-written as

$$
\begin{gathered}
\check{\gamma}_{k_{2}, c}=\min \left(\gamma_{1, k_{2}, c}, \gamma_{2, k_{2}, c}\right), \\
\check{\gamma}_{k_{2}, p}=\min \left(\gamma_{1, k_{2}, p}, \gamma_{2, k_{2}, p}\right),
\end{gathered}
$$

where $\gamma_{1, k_{2}, a}$ and $\gamma_{2, k_{2}, a}, a \in\{c, p\}$ are defined in (27) and (28), respectively. Substituting (39) in (30) followed by steps performed in (31)-(32), we can obtain the closed-form expression of end-to-end outage probability of $k_{2}$-th CEU (assisted by a DF-RS relay only) as

$$
\begin{aligned}
\dot{\mathbb{P}}_{k_{2}}^{\text {out,DF }} \triangleq 1-\exp [ & -\frac{\max \left(\delta_{1}\left(\tau_{c}\right), \delta_{2}\left(\tau_{p}\right)\right)}{\rho_{B}} \\
& \left.-\frac{\max \left(\delta_{3}\left(\tau_{c}\right), \delta_{4}\left(\tau_{p}\right)\right)}{\rho_{R}}\right],
\end{aligned}
$$

where $\delta_{i}(\cdot), i=1,2,3,4$, is same as given in (14) and (35).

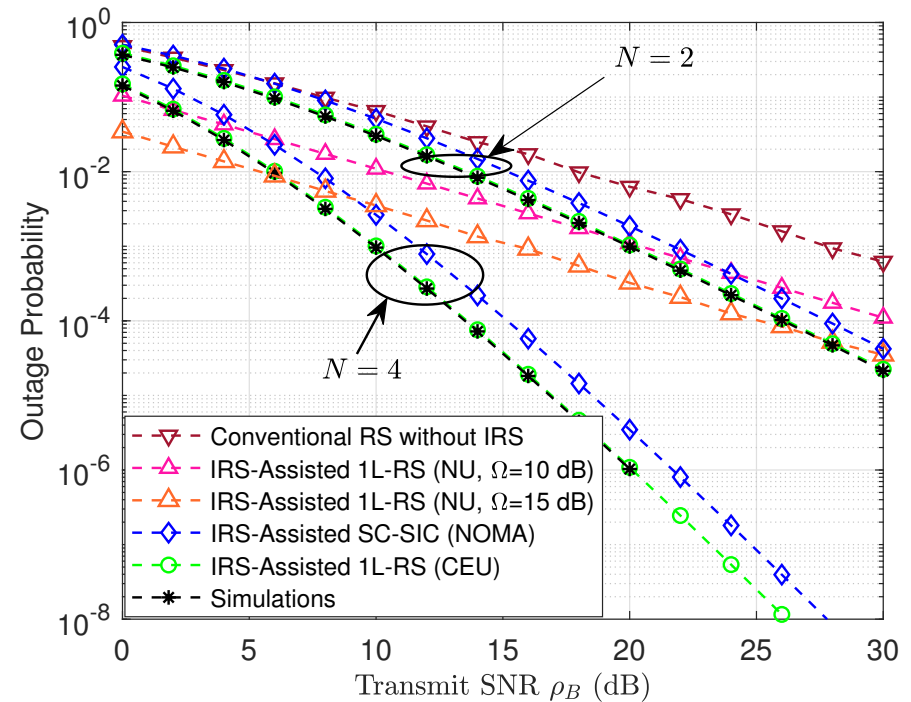

Fig. 4: Outage performance of IRS-assisted cell-edge user with RS based downlink environment having $K=2$ users.

\section{NUMERICAL RESULTS}

The simulation and analytical results for the outage behavior of IRS-assisted multi-user downlink communication system with RS are presented in this section. We adopt the IRS placement policy given in [9]. Accordingly, we deploy the IRSs near to cell-edge boundary such that only one CEU can hear from it's associated IRS. The average channel powers of BS-IRS and IRS-CEU links are assumed to be unity. Further, we have assumed the ideal reflection scenario at each IRS, i.e., the reflection amplitude of all the active reflecting elements is unity. Additionally, it is assumed that $40 \%$ of the total transmit power $\left(\mathcal{P}_{B}\right)$ is assigned to common data, i.e., $\alpha_{c}=0.4$. The remaining power is divided among all private messages such that $\sum_{k=1}^{K} \alpha_{k}=1-\alpha_{c}$. Throughout the numerical results, it is considered that $\bar{R}_{c}=\bar{R}_{p}=0.25 \mathrm{BPCU}$. Without loss of generality, we consider $K_{1}=K_{2}=0.5 K$, i.e., there are equal number of NUs and CEUs. However, our results are applicable to any arbitrary distribution of $K_{1}$ and $K_{2}$.

Fig. 4 shows the outage performance of the NU and the CEU in the IRS-assisted downlink two-user system utilizing 1L-RS at the BS. For the outage performance of NU, we assume $\Omega=$ 10,15 dB. It can be seen in Fig. 4 that the derived analytical results coincide with the simulated outage probability values, which confirms the accuracy of our analysis. It can be observed from Fig. 4 that for two-user scenario, the IRS-assisted CEU outperforms the NU beyond certain $\rho_{B}$. However, the SNR value beyond which the CEU outperforms the NU, depends on different operational conditions such as number of reflecting elements at IRS, number of NUs and CEUs in the system, average channel powers of BS-NU and BS-IRS-CEU links, target data rate, and the power allocation coefficients of the common and private messages, etc.

Example 1: Under single $N U$ and single $C E U$ case considered in Fig. 4 with BS-NU average channel power of 10 $d B$, the SNR value after which the CEU outperforms the NU, is $\rho_{B} \approx 20 d B$ for $N=2$, whereas for $N=4$, this value reduces to $\rho_{B} \approx 2 d B$. However, if we have $\Omega=15 d B$, the 


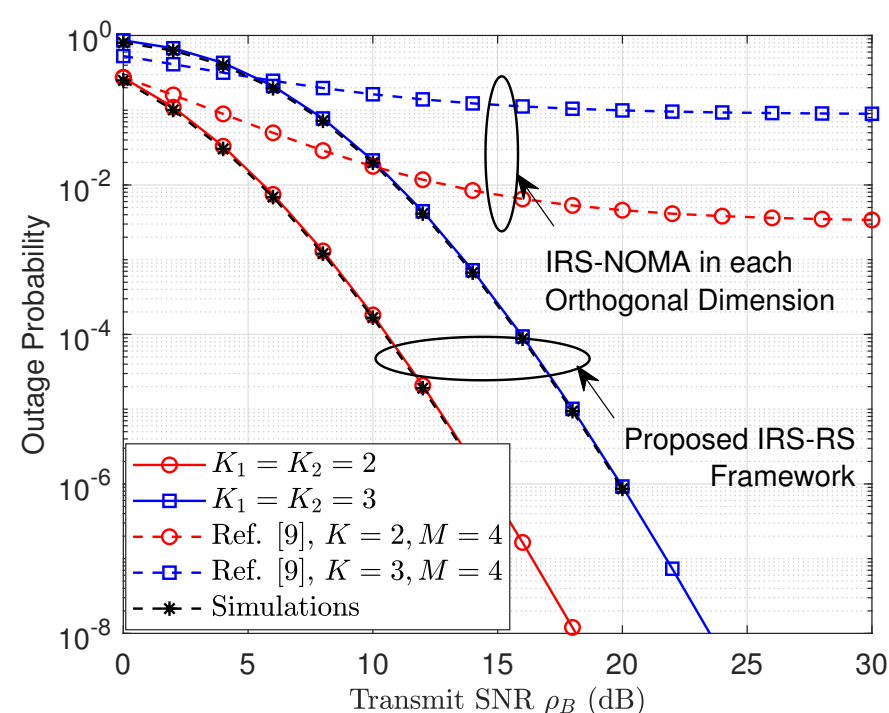

Fig. 5: Outage performance comparison of the proposed IRS-RS downlink communication system and IRS-NOMA framework [9] for $K=4,6$ users and $N=8$.

CEU outperforms the $N U$ after $\rho_{B} \approx 6 d B$ and $\rho_{B} \approx 27 d B$ for $N=2$ and $N=4$, respectively.

The results for the proposed IRS-assisted $1 \mathrm{~L}-\mathrm{RS}^{1}$ scheme are compared with IRS-assisted NOMA, where the BS utilizes power domain NOMA by combining all the user messages using superposition coding (SC). The communication between the BS and the CEU takes place through an IRS and the CEU performs SICs before decoding its own data. Fig. 4 demonstrates the superiority of the proposed IRS-assisted $1 \mathrm{~L}$ RS system over corresponding IRS-assisted NOMA framework $^{2}$ with fixed receiver complexity. The RS scheme achieves multiplexing gain advantage as compared to the NOMA scheme, however, the rate of decay of outage probability curves (i.e., the diversity order) remain same for the two schemes. The performance gain of 1L-RS is consistent with different values of $N$. Furthermore, the outage performance of the considered IRS-assisted RS framework is compared with the conventional RS framework without IRS, where the BS directly communicates with the CEUs using 1L-RS. Fig. 4 depicts that the use of IRS near to the CEU enhances the performance significantly and the performance gain increases drastically with the increase in $N$.

Fig. 5 shows the outage performance of IRS-assisted 1LRS scheme for four-user and six-user downlink scenarios, where number of cell-edge users are 2 and 3, respectively. The simulation results exactly matches with outage values obtained through derived analytical expressions for both the scenarios. Further, the outage behavior of the proposed IRSRS framework is compared with an IRS-assisted NOMA

\footnotetext{
${ }^{1}$ In RS, a fraction of the interference is decoded and the remaining fraction is treated as noise. Thus, RS serves as a soft bridge between SDMA (fully treats interference as noise) and NOMA (fully decodes interference) to achieve required QoS with low complexity designs.

${ }^{2}$ If we force one user to completely decode the other user message in a two-user downlink scenario, the RS reduces to NOMA. This can be achieved by encoding the second user message into common message and allocating zero power to the corresponding private message [34].
}

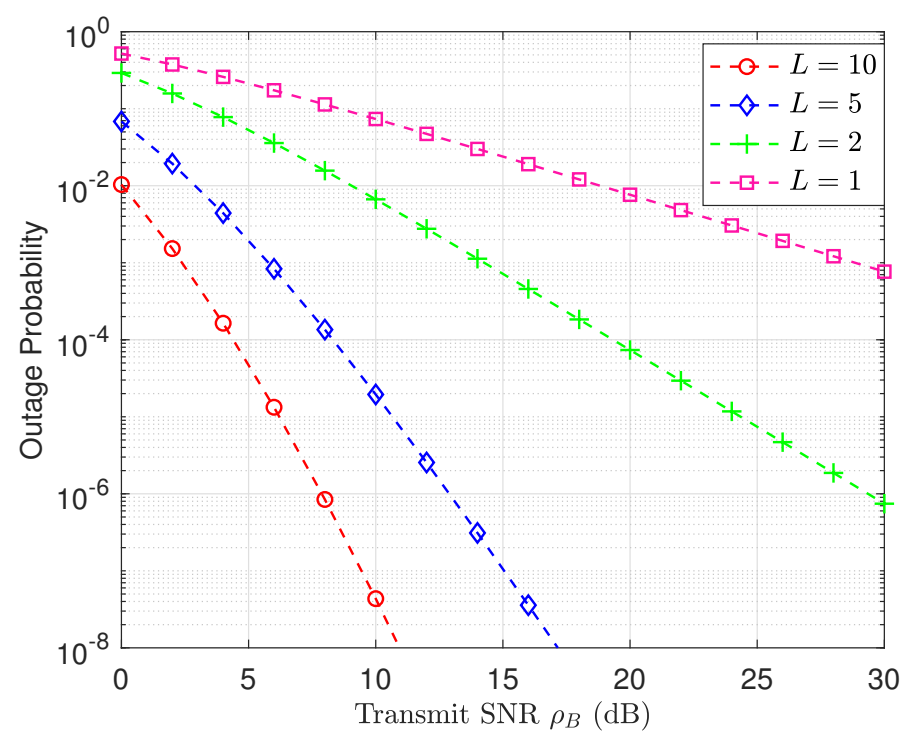

Fig. 6: Impact of $L$ on the outage performance of the proposed IRS-assisted RS framework for $K=2$ and $N=10$.

framework considered in [9], where the multiple antenna BS utilizes NOMA in each orthogonal spatial dimension to serve one NU and one IRS-assisted CEU. Both the considered IRSRS and IRS-NOMA frameworks employs on-off controlling scheme at the IRS.

It is clear from Fig. 5 that the proposed IRS-RS technique with single antenna BS outperforms the IRS-NOMA framework of [9] having multiple antenna BS (with $M=4$ ) by a huge margin. It can be noticed from Fig. 5 that the performance of IRS-NOMA framework with orthogonal user groups saturates at high transmit SNR for all the system parameters considered in the figure. However, the outage probability of the proposed IRS-RS system decays very rapidly under both four-user and six-user scenarios.

Example 2: It can be noted from Fig. 5 that under 6-user scenario, the outage probability values of the proposed IRS-RS framework at $20 d B$ and $22 d B$ are $9.236 \times 10^{-7}$ and $7.33 \times$ $10^{-8}$, respectively. Using the relation $d=-\lim _{\rho_{B} \rightarrow \infty} \frac{\log _{10} \mathbb{P}_{k_{2}} \times \log _{10}}{\log _{10} \rho_{B}}$, we get $d \approx 5.5$. Thus, it can be deduced that the proposed framework achieves a diversity order of approximately 5.5.

Fig. 6 illustrates the impact of $L$ on the outage performance of IRS-assisted 1L-RS framework with $K=2$ and $N=10$. It can be observed from Fig. 6 that for all the transmit SNR values, the outage performance improves with increasing $L$ and when $L=N$ we get the best outage performance. This is intuitive from (12) as each term in outage probability expression decreases with increasing value of exponent $L(\geq 1)$. It can also be deduced from the discussion given in Remark 1 that the best outage performance is achieved with maximum possible value of $L$, (i.e., $L=N$ ). In addition, it can be seen in Fig. 6 that for $L=5$, the outage probability reduces from $3.1 \times 10^{-7}$ to $3.58 \times 10^{-8}$ for $\rho_{B}$ increasing from $14 \mathrm{~dB}$ to 16 $\mathrm{dB}$ only. Whereas, for a transmit SNR increase of $2 \mathrm{~dB}$ (from $26 \mathrm{~dB}$ to $28 \mathrm{~dB}$ ) for $L=2$, the outage probability decays from $4.68 \times 10^{-6}$ to $1.87 \times 10^{-6}$ only. This clearly shows that the diversity gain improves significantly as $L$ increases. 


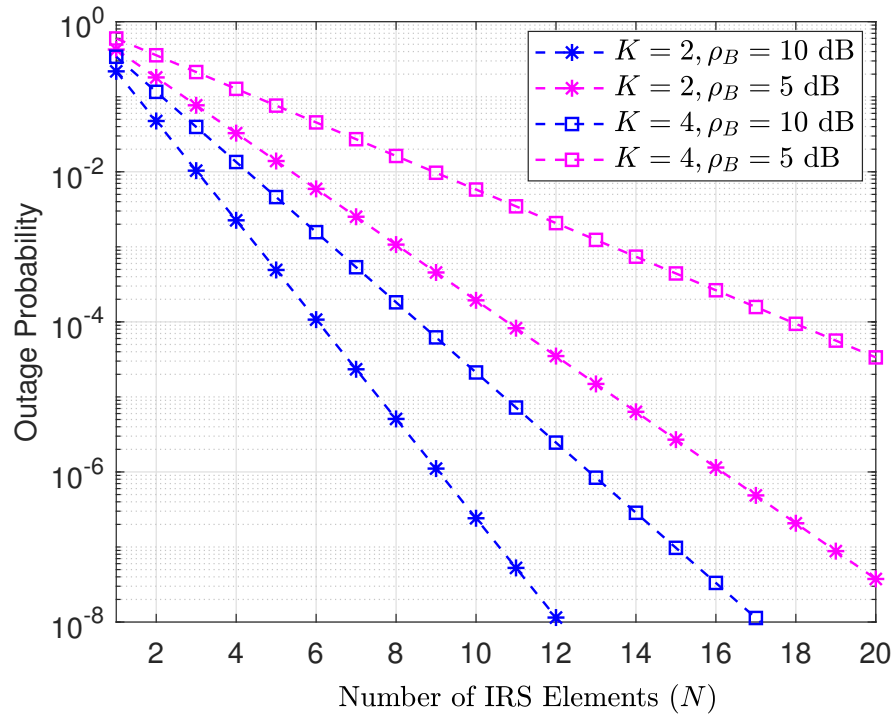

Fig. 7: Outage probability versus $N$ with $K=2,4$ and $\rho_{B}=5,10 \mathrm{~dB}$.

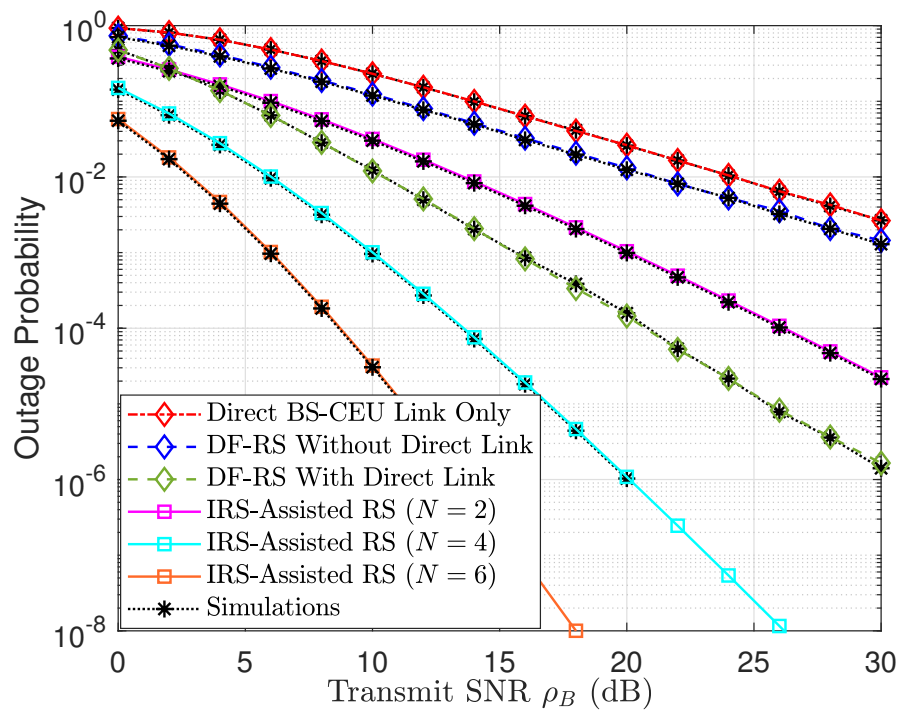

Fig. 8: Comparison of outage probability for a CEU under the proposed IRSRS and cooperative DF-RS frameworks with $\rho_{R}=\rho_{B}$.

In Fig. 7, we show the outage performance versus the number of IRS reflecting elements $N$ for two-user $(K=2)$ and four-user $(K=4)$ downlink scenarios with $\rho_{B}=5 \mathrm{~dB}$ and $10 \mathrm{~dB}$. It can be observed in Fig. 7 that we achieve the better performance gain with increasing $N$. The main reason behind this is that increasing the number of reflectors at the IRS provides more degrees of freedom (DoFs) for the signal transmitted by the BS. Furthermore, when we increase the number of users from $K=2$ to $K=4$, the performance deteriorates due to increase in interference power.

Fig. 8 demonstrates the comparison of CEU outage performance under the IRS-RS downlink network and the cooperative DF-RS communication network for $K=2$. We set $\alpha_{c}=0.4, \alpha_{2}=0.36$, and $\rho_{B}=\rho_{R}$. It can be observed from Fig. 8 that performance of an IRS-assisted CEU is superior as compared to the performance of a dual-hop DF relay-assisted $\mathrm{CEU}$ (without direct link between the BS and the CEU) for all

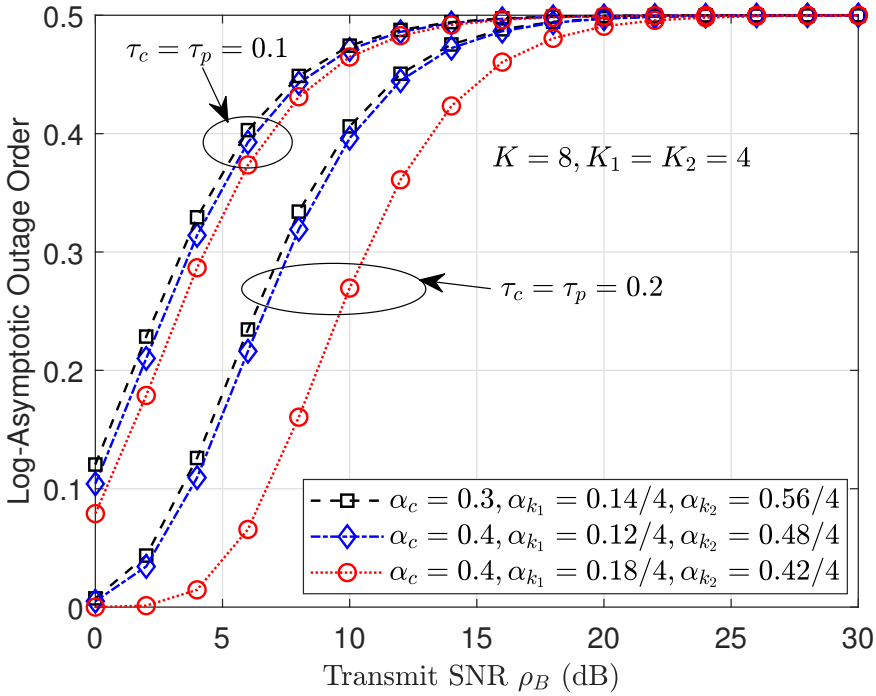

Fig. 9: Log Asymptotic Outage Order for a CEU under sufficiently large number of reflecting elements at IRS with 8 user scenario, i.e., 4 NUs and 4 CEUs.

values of $\rho_{B}$ and $N$ considered in the figure. If a direct BSCEU link is available (with average channel gain $\Omega^{\prime}=-6$ $\mathrm{dB}$ ) along with DF relay based cooperative link, the outage performance of CEU significantly improves and outperforms the IRS-assisted scenario for $N=2$. However, for $N>2$, the IRS-assisted CEU experiences substantial performance gain in comparison to DF relay based CEU with direct link. It can also be observed from Fig. 8 that the outage performance of CEU is worst in the case when the CEU is only receiving the signal through direct BS-CEU link. Simulation results for outage probability of DF relay-assisted framework corroborates the analytical results derived in Section IV.

Fig. 9 shows the variations of Log-Asymptotic Outage Order for large values of $N$ with respect to transmit SNR at the BS. For this figure, we consider a cellular network of 8 users with equal NUs and CEUs. We assume that power allocated to the private data stream of each CEU is same. We consider two different values of threshold SINRs as $\tau_{c}=\tau_{p}=0.1$ and $\tau_{c}=\tau_{p}=0.2$ along with $D=1$. In Fig. 9, the Log-Asymptotic Outage Order of $k_{2}$-th CEU, $k_{2}=K_{1}+1, K_{1}+2, \ldots, K$ is examined for different settings of common and private power allocation coefficients. It can be noticed from Fig. 9 that under considered parameter settings, $\mathcal{O}_{k_{2}, N}$ saturates at 0.5 for high transmit SNR. Furthermore, it can be observed from Fig. 9 that more power to CEU's private data stream provides better $\mathcal{O}_{k_{2}, N}$ performance for a fixed common data stream power under both the threshold conditions. However, if we reduce the common power by $10 \%$ of the total transmit power (i.e., $0.1 \mathcal{P}_{B}$ ) by maintaining a fixed ratio between $\alpha_{k_{1}}$ and $\alpha_{k_{2}}$, the Log-Asymptotic Outage Order increases. Thus, it can be deduced from Fig. 9 that the private data stream power allocation coefficient significantly controls the overall performance of the CEU, specially under the conditions of large number of reflecting elements.

Fig. 10 shows the impact of estimation errors of BS-IRS channel on the outage performance of the considered network 


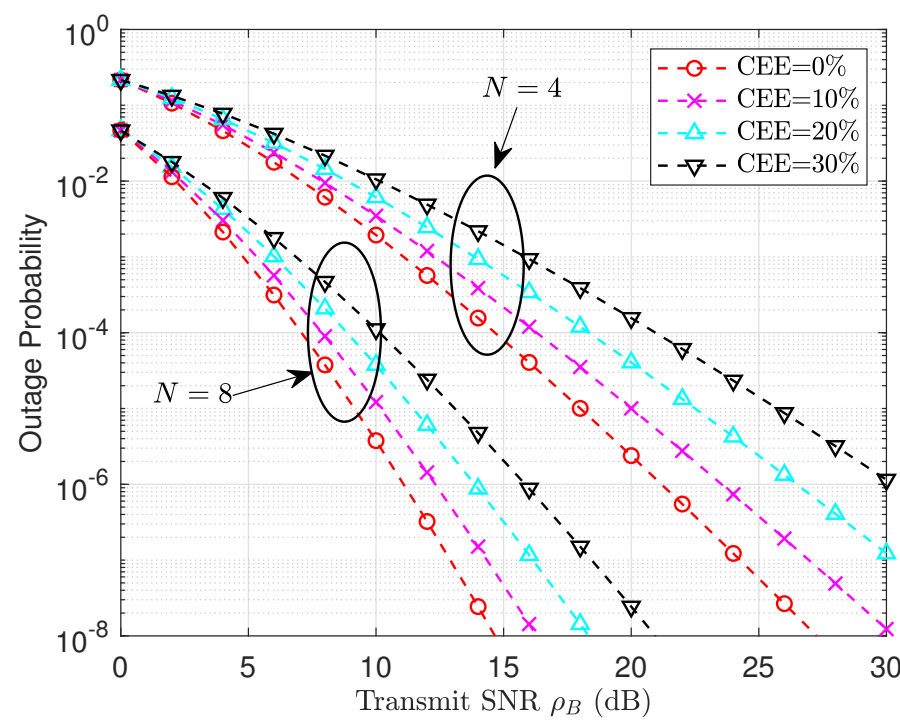

Fig. 10: Impact of channel estimation error of BS-IRS link on the outage performance of IRS-assisted CEU under 4 user scenario (2 NUs and 2 CEUs) and $N=4,8$.

under 4 user scenario $\left(K_{1}=K_{2}=2\right)$ for different values of $N$. We have assumed equal power allocation coefficient for common message and CEU private messages as $\alpha_{c}=$ $\alpha_{k_{2}}=0.25$. The channel estimation error (CEE) for BS$\operatorname{IRS}_{k_{2}}$ channel is defined as CEE $=\mathbb{E}\left[\left\|\mathbf{h}_{k_{2}}-\hat{\mathbf{h}}_{k_{2}}\right\|^{2}\right]$, where $\hat{\mathbf{h}}_{k_{2}}$ denotes the estimated channel vector corresponding to the exact channel vector $\mathbf{h}_{k_{2}}$. We have considered different values for percentage CEE of BS-IRS $k_{2}$ channel $(10 \%, 20 \%$, and $30 \%$ ) and have compared the outage performance under imperfect channel state information (CSI) scenario with the perfect CSI case (i.e., 0\% CEE). Although it is intuitive that the system performance will degrade under imperfect CSI scenario, however, it can be observed from Fig. 10 that the performance degradation for $N=8$ is small as compared to that for $N=4$. For example, to attain an outage probability of 0.0001 with $30 \%$ CEE, an additional transmit SNR (with respect to $0 \% \mathrm{CEE}$ ) of approximately $3 \mathrm{~dB}$ and $6 \mathrm{~dB}$ is required for $N=8$ and $N=4$, respectively. Moreover, it should be noted that the transmit SNR requirement further increases as we lower the allowable limit for outage probability.

\section{CONCLUSIONS}

This paper has proposed a novel IRS-RS framework to enhance the performance of multi-user downlink communication system by employing an IRS near to cell-edge users as well as $1 \mathrm{~L}-\mathrm{RS}$ at the BS. To analyze the system performance, we have derived the closed-form expressions of outage probability for the users. In addition, we also studied the performance of DF-RS framework. Next, through extensive simulation results, we have shown that with 1L-RS IRS-assisted downlink multiuser communication system gives better performance than that of the IRS-NOMA. Moreover, the performance of IRS-RS system has been also compared with the corresponding DF-RS framework. Furthermore, we have demonstrated the impact of BS-IRS channel estimation errors, employing higher number of IRS reflecting elements $N$ and the number of users $K$ on the system performance in terms of outage probability.

\section{APPENDIX A}

Since, the $\ell$-th row vector of $\mathbf{F}$ will have the following form

$$
\mathbf{f}_{\ell}=D^{-\frac{1}{2}}[\overbrace{0,0, \ldots, 0}^{(\ell-1) D \text { Terms }}, \overbrace{1,1, \ldots, 1}^{D \text { Terms }}, \overbrace{0,0, \ldots, 0}^{(L-\ell) D \text { Terms }}],
$$

the term $\mathbf{f}_{\ell} \mathbf{G}_{k_{2}} \mathbf{h}_{k_{2}}$ can be expressed as

$$
\begin{aligned}
\mathbf{f}_{\ell} \mathbf{G}_{k_{2}} \mathbf{h}_{k_{2}} & =D^{-\frac{1}{2}} \sum_{n=(\ell-1) D+1}^{\ell D} g_{k_{2}, n} h_{k_{2}, n}, \\
& \triangleq D^{-\frac{1}{2}} \sum_{d=1}^{D} g_{k_{2}, d} h_{k_{2}, d},
\end{aligned}
$$

where the second line utilizes $d=n-(\ell-1) D$. Considering a case with $L=2$ and $D=1$, let us find an $\mathbf{f}_{\ell}, \ell=1,2$ that maximizes the common SINR $\gamma_{k_{2}, c}\left(\mathbf{f}_{\ell}\right)$ as

$$
\gamma_{k_{2}, c}\left(\mathbf{f}_{1}\right) \underset{\mathbf{f}_{2}}{\stackrel{\mathbf{f}_{1}}{\gtrless}} \gamma_{k_{2}, c}\left(\mathbf{f}_{2}\right),
$$

Using (42) with $D=1$, we may write $\mathbf{f}_{1} \mathbf{G}_{k_{2}} \mathbf{h}_{k_{2}}=g_{k_{2}, 1} h_{k_{2}, 1}$ and $\mathbf{f}_{2} \mathbf{G}_{k_{2}} \mathbf{h}_{k_{2}}=g_{k_{2}, 2} h_{k_{2}, 2}$. Substituting the same in the expression of $\gamma_{k_{2}, c}\left(\mathbf{f}_{\ell}\right)$ (given in (8)), we can rewrite (43) as

$$
\left|g_{k_{2}, 1} h_{k_{2}, 1}\right|{\underset{\mathbf{f}}{\mathbf{f}_{2}}}_{\mathbf{f}_{1}}^{\sum_{1}}\left|g_{k_{2}, 2} h_{k_{2}, 2}\right| .
$$

Similarly, on obtaining a condition for the maximization of private SINR $\gamma_{k_{2}, p}\left(\mathbf{f}_{\ell}\right)$, we again (44). This implies that both common and private SINRs are maximized by same $\mathbf{f}_{\ell}$. Following similar steps, we generalize the condition in (44) for arbitrary $L$ and $D=1$ as

$$
\ell^{*}=\underset{\ell=1,2, \ldots, L}{\arg \max }\left|g_{k_{2}, \ell} h_{k_{2}, \ell}\right|,
$$

such that resulting $\mathbf{f}_{\ell^{*}}$ maximizes both common as well as private SINRs. Now, consider another case of $L=2$ and $D=2$. Using (42), we may write

$$
\begin{aligned}
\mathbf{f}_{1} \mathbf{G}_{k_{2}} \mathbf{h}_{k_{2}} & =\frac{1}{\sqrt{2}}\left(g_{k_{2}, 1} h_{k_{2}, 1}+g_{k_{2}, 2} h_{k_{2}, 2}\right), \\
\mathbf{f}_{2} \mathbf{G}_{k_{2}} \mathbf{h}_{k_{2}} & =\frac{1}{\sqrt{2}}\left(g_{k_{2}, 3} h_{k_{2}, 3}+g_{k_{2}, 4} h_{k_{2}, 4}\right) .
\end{aligned}
$$

Using (46) in (8) for both common and private SINRs, we again get the same result as

$$
\left|g_{k_{2}, 1} h_{k_{2}, 1}+g_{k_{2}, 2} h_{k_{2}, 2}\right| \sum_{\mathbf{f}_{2}}^{\mathbf{f}_{1}}\left|g_{k_{2}, 3} h_{k_{2}, 3}+g_{k_{2}, 4} h_{k_{2}, 4}\right|,
$$

which again verifies that same row vector $\mathbf{f}_{\ell}$ maximizes both the SINRs given in (8). Based on these observations, we finally generalize the condition for arbitrary $L$ and $D$ as

$$
\ell^{*}=\underset{\ell=1,2, \ldots, L}{\arg \max }\left|\sum_{n=(\ell-1) D+1}^{\ell D} g_{k_{2}, n} h_{k_{2}, n}\right| .
$$

Thus, it is clear from the above discussion that a row vector with index $\ell^{*}$ will always maximize both common and private SINRs of a CEU, where $\ell^{*}$ is obtained by following (48). 


\section{APPENDIX B \\ PROOF OF LEMMA 1}

If $\mathbf{f}_{\ell} \mathbf{G}_{k_{2}} \mathbf{h}_{\mathbf{k}_{\mathbf{2}}} \triangleq Z_{\ell}$ and $\left\{h_{k_{2}, d}\right\}_{d=1}^{D}$ are known, it immediately follows that

$$
\left.Z_{\ell}\right|_{\left\{h_{k_{2}, d}\right\}_{d=1}^{D}} \sim \mathcal{C N}\left(0, D^{-1} \sum_{d=1}^{D}\left|h_{k_{2}, d}\right|^{2}\right) .
$$

and

$$
\left.\left|Z_{\ell}\right|^{2}\right|_{\left\{h_{k_{2}, d}\right\}_{d=1}^{D}} \sim \operatorname{Exp}\left(D^{-1} \sum_{d=1}^{D}\left|h_{k_{2}, d}\right|^{2}\right) .
$$

Since $h_{k_{2}, d} \sim \mathcal{C N}(0,1)$, the $\mathrm{RV} W_{\ell} \triangleq D^{-1} \sum_{d=1}^{D}\left|h_{k_{2}, d}\right|^{2}$ follows Chi-square distribution with $2 D$ DoFs. The probability density function (PDF) of $W_{\ell}$ can be given as

$$
f_{W_{\ell}}(w)=\frac{D^{D}}{\Gamma(D)} e^{-D w} w^{D-1} \quad w \geq 0 .
$$

Thus, the PDF of $\left|Z_{\ell}\right|^{2}$ can be evaluated using (51) in $f_{\left|Z_{\ell}\right|^{2}}(z)=\int_{0}^{\infty} \frac{1}{w} e^{-\frac{z}{w}} f_{W_{\ell}}(w) d w$ followed by the use of [33, Eq. 3.471.9] as

$$
f_{\left|Z_{\ell}\right|^{2}}(z)=\frac{2 D^{\frac{D+1}{2}}}{\Gamma(D)} z^{\frac{D-1}{2}} K_{D-1}(2 \sqrt{D z}) .
$$

The CDF $\mathbb{F}_{\left|Z_{\ell}\right|^{2}}(z)=\int_{0}^{z} f_{\left|Z_{\ell}\right|^{2}}(v) d v$, can be obtained by using (52) and [33, Eq. 6.561.8] as

$$
\mathbb{F}_{\left|Z_{\ell}\right|^{2}}(z)=1-\frac{2}{\Gamma(D)}(D z)^{\frac{D}{2}} K_{D}(2 \sqrt{D z}) .
$$

Substituting $\mathbf{f}_{\ell} \mathbf{G}_{k_{2}} \mathbf{h}_{\mathbf{k}_{\mathbf{2}}} \triangleq Z_{\ell}$ in (8), we may now write the joint CDF of $\gamma_{k_{2}, c}\left(\mathbf{f}_{\ell}\right)$ and $\gamma_{k_{2}, p}\left(\mathbf{f}_{\ell}\right)$ as

$$
\begin{aligned}
& \mathbb{F}_{\gamma_{k_{2}, c}\left(\mathbf{f}_{\ell}\right), \gamma_{k_{2}, p}\left(\mathbf{f}_{\ell}\right)}(\mu, \nu) \\
& =\operatorname{Pr}\left\{\frac{\alpha_{c}\left|Z_{\ell}\right|^{2}}{\sum_{k=1}^{K} \alpha_{k}\left|Z_{\ell}\right|^{2}+\frac{1}{\rho_{B}}} \leq \mu, \frac{\alpha_{k_{2}}\left|Z_{\ell}\right|^{2}}{\sum_{\substack{k=1 \\
k \neq k_{2}}}^{K} \alpha_{k}\left|Z_{\ell}\right|^{2}+\frac{1}{\rho_{B}}} \leq \nu\right\}, \\
& =\operatorname{Pr}\left\{\left|Z_{\ell}\right|^{2} \leq \min \left(\frac{\delta_{1}(\mu)}{\rho_{B}}, \frac{\delta_{2}(\nu)}{\rho_{B}}\right)\right\}, \\
& =\mathbb{F}_{\left|Z_{\ell}\right|^{2}}\left(\min \left(\frac{\delta_{1}(\mu)}{\rho_{B}}, \frac{\delta_{2}(\nu)}{\rho_{B}}\right)\right) .
\end{aligned}
$$

where $\delta_{1}(\mu)$ and $\delta_{2}(\nu)$ are defined in (14). Using (53) in (54) and substituting $\mu=\tau_{c}$ and $\nu=\tau_{p}$, we get (13).

\section{REFERENCES}

[1] M. D. Renzo, M. Debbah, D.-T. Phan-Huy et al., "Smart radio environments empowered by reconfigurable AI meta-surfaces: An idea whose time has come," J. Wireless Commun. Netw., May 2019, Article number: 129 (2019)

[2] Q. Wu and R. Zhang, "Towards smart and reconfigurable environment: Intelligent reflecting surface aided wireless network," IEEE Commun. Mag., vol. 58, no. 1, pp. 106-112, Jan. 2020.

[3] K. Singh, A. Gupta, and T. Ratnarajah, "Energy efficient resource allocation for multiuser relay networks," IEEE Trans. Wireless Commun., vol. 16, no. 2, pp. 1218-1235, Feb. 2017.
[4] K. Singh, A. Gupta, and T. Ratnarajah, "A utility-based joint subcarrier and power allocation for green communications in multi-user two-way regenerative relay networks," IEEE Trans. Commun., vol. 65, no. 9, pp. 3705-3722, Sept. 2017.

[5] K. Singh, A. Gupta, T. Ratnarajah, and M.-L. Ku, "A general approach toward green resource allocation in relay-assisted multiuser communication networks," IEEE Trans. Wireless Commun., vol. 17, no. 2, pp. 848-862, Feb. 2018.

[6] L.-A. Matti and L. Kari, "Key drivers and research challenges for 6G ubiquitous wireless intelligence," Sept. 2019, [Online]. Available: http://jultika.oulu.fi/files/isbn9789526223544.pdf.

[7] E. Basar, "Reconfigurable intelligent surface-based index modulation: A new beyond MIMO paradigm for 6G," IEEE Trans. Commun., vol. 68 , no. 5, pp. 3187-3196, May 2020.

[8] E. Basar, M. Di Renzo, J. De Rosny, M. Debbah, M. Alouini, and R. Zhang, "Wireless communications through reconfigurable intelligent surfaces," IEEE Access, vol. 7, pp. 116753-116773, Aug. 2019.

[9] Z. Ding and H. Vincent Poor, "A simple design of IRS-NOMA transmission," IEEE Commun. Lett., vol. 24, no. 5, pp. 1119-1123, May 2020.

[10] G. Zhou, C. Pan, H. Ren, K. Wang, M. D. Renzo, and A. Nallanathan, "Robust beamforming design for intelligent reflecting surface aided MISO communication systems," IEEE Wireless Commun. Lett., vol. 9, no. 10, pp. 1658-1662, Oct. 2020 .

[11] W. Huang, Y. Zeng, and Y. Huang. "Achievable rate region of MISO interference channel aided by intelligent reflecting surface," IEEE Trans. Veh. Technol., vol. 69, no. 12, pp. 16264-16269, Dec. 2020.

[12] W. Fang, M. Fu, Y. Shi, and Y. Zhou, "Outage minimization for intelligent reflecting surface aided MISO communication systems via stochastic beamforming," in Proc. IEEE SAM, Hangzhou, China, Jun. 2020, pp. $1-5$.

[13] X. Yu, D. Xu, D. W. K. Ng, and R. Schober, "Power-efficient resource allocation for multiuser MISO systems via intelligent reflecting surfaces," in Proc. IEEE GLOBECOM, Taipei, Taiwan, Dec. 2020, pp. 1-6.

[14] E. Björnson, Ö. Özdogan, and E. G. Larsson, "Intelligent reflecting surface versus decode-and-forward: How large surfaces are needed to beat relaying?," IEEE Wireless Commun. Lett., vol. 9, no. 2, pp. 244-248, Feb. 2020.

[15] M. Hua, Q. Wu, D. W. K. Ng, J. Zhao, and L. Yang, "Intelligent reflecting surface-aided joint processing coordinated multipoint transmission," IEEE Trans. Commun., vol. 69, no. 3, pp. 1650-1665, Mar. 2021.

[16] Y. Guo, Z. Qin, Y. Liu, and N. Al-Dhahir, "Intelligent reflecting surface aided multiple access over fading channels," IEEE Trans. Commun., vol. 69, no. 3, pp. 2015-2027, Mar. 2021.

[17] Y. Liu, J. Zhao, M. Li, and Q. Wu, "Intelligent reflecting surface aided MISO uplink communication network: Feasibility and power minimization for perfect and imperfect CSI," IEEE Trans. Commun., vol. 69, no. 3, pp. 1975-1989, Mar. 2021.

[18] B. Clerckx, H. Joudeh, C. Hao, M. Dai and B. Rassouli, "Rate splitting for MIMO wireless networks: A promising PHY-layer strategy for LTE evolution," IEEE Commun. Mag., vol. 54, no. 5, pp. 98-105, May 2016.

[19] H. Joudeh and B. Clerckx, "Sum-rate maximization for linearly precoded downlink multiuser MISO systems with partial CSIT: A rate-splitting approach," IEEE Trans. Commun., vol. 64, no. 11, pp. 4847-4861, Nov. 2016.

[20] H. Joudeh and B. Clerckx, "Robust transmission in downlink multiuser MISO systems: A rate-splitting approach," IEEE Trans. Signal Process., vol. 64, no. 23, pp. 6227-6242, Dec. 2016.

[21] Y. Mao, B. Clerckx, and V. O. K. Li, "Rate-splitting multiple access for downlink communication systems: Bridging, generalizing, and outperforming SDMA and NOMA," J. Wireless Com. Network, May 2018, Article number: 133 (2018).

[22] M. Dai, B. Clerckx, D. Gesbert and G. Caire, "A Rate Splitting Strategy for Massive MIMO With Imperfect CSIT," IEEE Trans. Wireless Commun., vol. 15, no. 7, pp. 4611-4624, Jul. 2016.

[23] J. Zhang, J. Zhang, Y. Zhou, H. Ji, J. Sun, and N. Al-Dhahir, "Energy and spectral efficiency tradeoff via rate splitting and common beamforming coordination in multicell networks," IEEE Trans. Commun., vol. 68, no. 12, pp. 7719-7731, Dec. 2020

[24] L. Yin and B. Clerckx, "Rate-splitting multiple access for multigroup multicast and multibeam satellite systems," IEEE Trans. Commun., vol. 68, no. 12 , pp. 7719-7731, Dec. 2020

[25] A. R. Flores, R. C. de Lamare, and B. Clerckx, "Linear precoding and stream combining for rate splitting in multiuser MIMO systems," IEEE Commun. Lett., vol. 24, no. 4, pp. 890-894, Apr. 2020.

[26] F. Fang, Y. Xu, Q. -V. Pham, and Z. Ding, "Energy-efficient design of IRS-NOMA networks," IEEE Trans. Veh. Technol., vol. 69, no. 11, pp. 14088-14092, Nov. 2020. 
[27] K. Singh et al., "Resource optimization in full duplex non-orthogonal multiple access systems," IEEE Trans. Wireless Commun., vol. 18, no. 9, pp. 4312-4325, Sept. 2019

[28] J. Zhang, B. Clerckx, J. Ge, and Y. Mao, "Cooperative rate splitting for MISO broadcast channel with user relaying, and performance benefits over cooperative NOMA," IEEE Signal Process. Lett., vol. 26, no. 11, pp. 1678-1682, Nov. 2019.

[29] P. Li, M. Chen, Y. Mao, Z. Yang, B. Clerckx, and M. Shikh-Bahaei, "Cooperative rate-splitting for secrecy sum-rate enhancement in multiantenna broadcast channels," in Proc. IEEE PIMRC, London, United Kingdom, Aug.-Sept. 2020, pp. 1-6.

[30] Y. Mao, B. Clerckx, J. Zhang, V. O. K. Li, and M. A. Arafah, "Max-min fairness of $K$-user cooperative rate-splitting in MISO broadcast channel with user relaying," IEEE Trans. Wireless Commun., vol. 19, no. 10, pp. 6362-6376, Oct. 2020.

[31] A. Z. Yalçın and Y. Yapıc1, "Max-min fair beamforming for cooperative multigroup multicasting with rate-splitting," IEEE Trans. Wireless Commun., vol. 20, no. 1, pp. 254-268, Jan. 2021.

[32] A. Papoulis and S. U. Pillai, Probability, Random Variables, and Stochastic Processes, 4th ed. McGraw-Hill, 2002.

[33] I. S. Gradshteyn and I. M. Ryzhik, Table of Integrals, Series and Products, 6th ed. New York: Academic Press, 2000.

[34] B. Clerckx, Y. Mao, R. Schober, and H. V. Poor, "Rate-splitting unifying SDMA, OMA, NOMA, and multicasting in MISO broadcast channel: A simple two-user rate analysis," IEEE Wireless Commun. Lett., vol. 9, no 3, Mar. 2020.

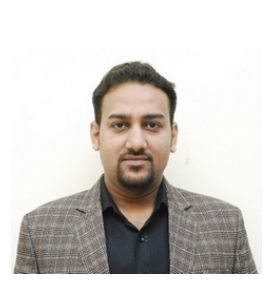

Ankur Bansal (Member, IEEE) received his BE degree in Electronics and Communication Engineering from RGPV University, Bhopal, India in 2010, M-Tech degree in ME and VLSI Design from MNNIT Allahabad, Prayagraj, India in 2013 and $\mathrm{Ph} . \mathrm{D}$. degree in Electronics and Communication Engineering from VNIT Nagpur, India in 2020. He is currently a post-doctoral researcher at the Institute of Communications Engineering, National Sun Yat-sen University (NSYSU), Taiwan. His current research duplex radio, OFDM, unmanned aerial vehicles.

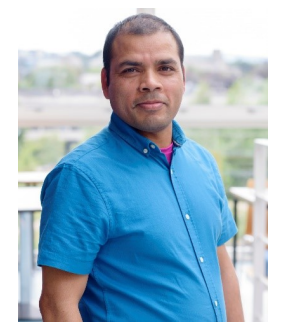

Keshav Singh (Member, IEEE) received the Master of Technology degree in computer science from Devi Ahilya Vishwavidyalaya, Indore, India, in 2006, the M.Sc. degree in information and telecommunications technologies from Athens Information Technology, Greece, in 2009, and the Ph.D. degree in communication engineering from National Central University, Taiwan, in 2015. He currently works at the Institute of Communications Engineering, National Sun Yatsen University (NSYSU), Taiwan as an Assistant Professor. Prior to this, he held the position of Research Associate from 2016 to 2019 at the Institute of Digital Communications, University of Edinburgh, U.K. From 2019 to 2020, he was associated with the University College Dublin, Ireland as a Research Fellow. He leads research in the areas of green communications, resource allocation, full-duplex radio, ultra-reliable low-latency communication, non-orthogonal multiple access, wireless edge caching, machine learning for communications, and large intelligent surface assisted communications.

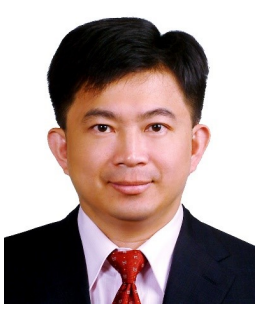

Chih-Peng Li (Fellow, IEEE) received the B.S. degree in Physics from National Tsing Hua University, Hsin Chu, Taiwan, in June 1989 and the $\mathrm{Ph} . \mathrm{D}$. degree in Electrical Engineering from Cornell University, Ithaca, NY, USA, in December 1997.

From 1998 to 2000, Dr. Li was a Member of Technical Staff with the Lucent Technologies. From 2001 to 2002, he was a Manager of the Acer Mobile Networks. In 2002, he joined the faculty of the Institute of Communications Engineering, National Sun Yat-sen University (NSYSU), Taiwan, as an assistant professor. He has been promoted to Full Professor in 2010. Dr. Li served as the Chairman of the Department of Electrical Engineering with NSYSU from 2012 to 2015. He was the Director of the Joint Research and Development Center of NSYSU and Brogent Technologies from 2015 to 2016. Dr. Li served as the Vice President of General Affairs with NSYSU from 2016 to 2017. He is currently the Dean of Engineering College with NSYSU. His research interests include wireless communications, baseband signal processing, and data networks.

Dr. Li is currently the Chair of the IEEE Broadcasting Technology Society Tainan Section. Dr. Li also serves as the Editor of the IEEE Transactions on Wireless Communications, the Associate Editor of the IEEE Transactions on Broadcasting, the General Co-Chair of IEEE Information Theory Workshop 2017, and the Member of Board of Governors with IEEE Tainan Section. Dr. $\mathrm{Li}$ was the lead guest editor of the Special Issue of International Journal of Antennas and Propagation. He was also the recipient of the 2014 Outstanding Electrical Engineering Professor Award of the Chinese Institute of Electrical Engineering Kaohsiung Section and the 2015 Outstanding Engineering Professor Award of the Chinese Institute of Engineers Kaohsiung Section.

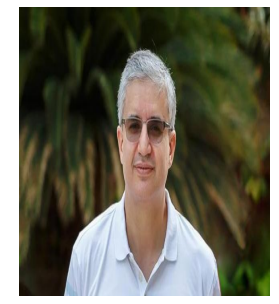

Mohamed-Slim Alouini (Fellow, IEEE) was born in Tunis, Tunisia. He received the Ph.D. degree in Electrical Engineering from the California Institute of Technology (Caltech), Pasadena, CA, USA, then in the Texas A\&M University at Qatar, Education City, Doha, Qatar before joining King Abdullah University of Science and Technology (KAUST), Thuwal, Makkah Province, Saudi Arabia as a Professor of Electrical Engineering in 2009. His current research interests include modeling, design, and performance analysis of wireless communication systems. 